\title{
Heterogeneous structure of poly(vinyl chloride) as the origin of anomalous dynamical behavior
}

\author{
A. Arbe \\ Unidad de Física de Materiales (CSIC-UPV/EHU), Apartado 1072, 20080 San Sebastián, Spain \\ A. Moral \\ Departamento de Física de Materiales, Universidad del País Vasco, Apartado 1072, \\ 20080 San Sebastián, Spain \\ A. Alegría \\ Unidad de Física de Materiales (CSIC-UPV/EHU), Apartado 1072, 20080 San Sebastián, Spain \\ and Departamento de Física de Materiales, Universidad del País Vasco, Apartado 1072, \\ 20080 San Sebastián, Spain \\ J. Colmenero \\ Unidad de Física de Materiales (CSIC-UPV/EHU), Apartado 1072, 20080 San Sebastián, Spain \\ and Departamento de Física de Materiales, Universidad del País Vasco, Apartado 1072, \\ 20080 San Sebastián, Spain and Donostia International Physics Center, Apartado 1072, 20080 San \\ Sebastián, Spain \\ W. Pyckhout-Hintzen and D. Richter \\ Institut für Festkörperforschung, Forschungszentrum Jülich, 52425 Jülich, Germany \\ B. Farago and B. Frick \\ Institut Laue-Langevin, 156X, 38042 Grenoble Cedex, France
}

(Received 6 February 2002; accepted 22 April 2002)

\begin{abstract}
We have investigated the thermal evolution of the structure and the dynamics of poly(vinyl chloride) $(\mathrm{PVC})$ in a wide temperature range. Corroborating earlier findings, small angle neutron scattering revealed the presence of structural heterogeneities. On the other hand, the single chain form factor corresponds to that of Gaussian chains. Gradually with increasing temperature the system becomes homogeneous. A simple description of the heterogeneities in terms of microcrystallites is forwarded. The dynamical behavior of PVC has been investigated combining broadband dielectric spectroscopy (DS) with coherent and incoherent neutron scattering. In a wide temperature range broadband DS facilitated a precise determination of the dynamic response related to the segmental relaxation. Close to the glass transition temperature the line shape strongly deviates from the usual Kohlrausch-Williams-Watts functional form of common glassforming systems. Moreover, the characteristic relaxation time observed by incoherent scattering displays an anomalous dependence on momentum transfer indicating the possible existence of heterogeneities in the sample. Based on the structural and dynamical results, a model is proposed, that considers the coexistence of regions with different dynamical properties leading to a distribution of characteristic relaxation times. The model accounts for the experimental observations, assuming for all regions the same functional form for the $\alpha$-relaxation. It may be univocally determined from the coherent scattering data at the first static structure peak. The distribution of relaxation times found is compatible with the distribution of only one variable, the glass transition temperature. (C) 2002 American Institute of Physics.
\end{abstract}

[DOI: $10.1063 / 1.1485283$ ]

\section{INTRODUCTION}

From a chemical point of view, poly(vinyl chloride) (PVC) is one of the simplest polymers, with a composition close to that of polyethylene. PVC is widely used, and its study also presents some practical interest. For both reasons, a large number of studies on the structure and dynamics of PVC can be found in the literature. ${ }^{1,2}$ The nature of this polymer has revealed to be so particular that even books have been devoted to it. ${ }^{2}$ Despite the big effort made, some of its peculiarities-well established since many years-are not yet understood. These concern structural as well as dynamical properties. A strong controversy on the "crystalline" nature of this polymer is still open. Evidences of heterogeneous morphology have been reported, ${ }^{3-9}$ but a clear interpretation of the structure is lacking. ${ }^{1,10,11}$ On the other hand, PVC undergoes a glass transition and displays dynamical properties like the $\alpha$ - and $\beta$-relaxations characteristic for glassforming systems. Already 60 years ago it was established that the $\alpha$-relaxation in PVC shows a rapidly increasing broadening with decreasing temperature approaching the glass transition temperature $T_{g} .{ }^{12}$ In 1967, McCrum et al. stated in their book: " "This effect (seen already in 1941 by Fuoss and Kirkwood ${ }^{12}$ ) appears to be characteristic of partially crystalline polymers such as PVC. It might result from 
the heterogeneity in structure of these systems which could give rise to a range of barrier heights or distribution of activation energies." However, this hypothesis has never been quantitatively checked. The reasons might be (i) the dielectric measurements were usually restricted to relatively low frequencies, preventing a complete characterization of the dielectric response at high temperatures; therefore, the temperature dependence of the $\alpha$-relaxation was known in only a narrow range; (ii) the lack of microscopic information in order to characterize the structural/dynamical heterogeneities. Since it couples directly to the density, neutron scattering is the appropriate tool in order to access such information. Concerning PVC structure, some experimental results obtained by small angle neutron scattering (SANS) have been reported, ${ }^{5,7}$ but they correspond to only one temperature.

In this work we have made an effort to push the available techniques in order to facilitate the study of the high temperature response of PVC. One of the techniques used is broad band dielectric spectroscopy (DS) covering a wide frequency range up to the $\mathrm{GHz}$ regime. This allows to characterize the response in a very large temperature range. Other than DS, in a complementary fashion quasielastic neutron scattering (NS) accesses the dynamical processes at a molecular level in space and time and offers direct microscopic insight into the molecular processes. In particular, the time evolution of the interchain correlations can be accessed in studying the coherent dynamic structure factor in the neighborhood of its first peak. Such experiments reveal the structural relaxation isolated from contributions from secondary relaxations. This allows the precise determination of the spectral shape of the $\alpha$-relaxation. Moreover, additional information on the existence of dynamical heterogeneities in the sample is offered by the $Q$-dependence of the incoherent scattering function. The combination of relaxation and NS techniques has already proven to be very successful for the investigation of the dynamics in several glass forming polymer systems. ${ }^{14-18}$

The goal of this work was to understand the peculiarities of the PVC dynamics in terms of an underlying heterogeneous structure, as it was suggested a long time ago. To do so we have first characterized the structural heterogeneities by studying the chain form factor and the structure factor at small angles. Furthermore the thermal evolution of the heterogeneities has been determined. Based on these results, a model is proposed that considers the presence of a distribution of regions with different structural relaxations. The observed distribution of characteristic times is compatible with the existence of only one distributed variable in the system, the glass transition temperature.

The paper is organized as follows: after the Experiment, where the sample characterization and the experimental techniques are explained, we present our SANS results on the structure of PVC. Thereafter, the dynamics results obtained by the different techniques are analyzed in the standard way for polymers. In the Discussion, the structural data are interpreted considering the presence of microcrystallites. Moving to the dynamics, first the anomalies compared to standard polymer dynamics are identified. Their possible sources are discussed. A model based on the heterogeneous nature of PVC is proposed and successfully applied to all dynamical data. Finally, the conclusions are presented.

\section{EXPERIMENT}

\section{A. Samples}

Since we were interested in both, incoherent and coherent scattering, we used protonated poly(vinyl chloride) (PVCh, $\left[-\mathrm{CH}_{2}-\mathrm{CHCl}-\right]_{n}$ ) and fully deuterated poly(vinyl chloride) (PVCd, $\left.\left[-\mathrm{CD}_{2}-\mathrm{CDCl}-\right]_{n}\right)$. Due to the different neutron scattering lengths of the isotopes $\mathrm{H}$ and $\mathrm{D}$, the dominant contribution to the measured scattering for PVCh is the incoherent scattering from the protons in the sample (the incoherent scattering cross section of hydrogen, $\sigma_{\mathrm{H}}^{\text {inc }}$ is much higher than any other cross section-coherent or incoherent - of the rest of the nuclei composing $\mathrm{PVCh}$ ). On the contrary, for PVCd the coherent scattering determines the measured intensities $\left(\sigma_{\mathrm{D}}^{\mathrm{coh}} \approx \sigma_{\mathrm{C}}^{\mathrm{coh}} \approx \sigma_{\mathrm{Cl}}^{\mathrm{coh}} / 3\right.$ are much higher than their incoherent counterparts).

The samples were synthesized by the group of Professor C. Mijangos (ICTP, CSIC, Madrid, Spain). Further details on the polymerization procedure can be found elsewhere. ${ }^{19}$ The values of the number-average molecular weight $M_{n}$ and the polydispersity $M_{w} / M_{n}$ of both samples were similar $\left(M_{n}\right.$ $\left.\cong 35 \times 10^{3}, M_{w} / M_{n} \cong 2\right)$. Also the tacticity, determined by nuclear magnetic resonance, was nearly the same (32\% syndiotactic, $50 \%$ hetereotactic, and $18 \%$ isotactic triads). The onset temperature for degradation $T_{\mathrm{deg}}$ of the PVCd sample was determined by a standard thermogravimetric test at a rate of $10 \mathrm{~K} / \mathrm{min}$. It revealed $T_{\mathrm{deg}}=596 \mathrm{~K}$, about $50 \mathrm{~K}$ higher than that obtained in the protonated case. The glass transition temperatures $T_{g}$ were determined by differential scanning calorimetry at a heating rate of $10 \mathrm{~K} / \mathrm{min}\left[T_{g}(\mathrm{PVCd})\right.$ $=358 \mathrm{~K}$ and $\left.T_{g}(\mathrm{PVCh})=353 \mathrm{~K}\right]$. In the temperature range $370 \mathrm{~K} \leqslant T \leqslant 420 \mathrm{~K}$, the mass density $d(T)$ varies with temperature like $d(T)\left(\mathrm{g} / \mathrm{cm}^{3}\right)=1.394-2.03 \times 10^{-4}(T[\mathrm{~K}]$ $-273)-2.19 \times 10^{-6}(T[\mathrm{~K}]-273)^{2} .^{20,21}$

\section{B. Broadband dielectric spectroscopy}

Dielectric measurements were performed on the protonated sample in the frequency domain from $10^{-2} \mathrm{~Hz}$ to $10^{9} \mathrm{~Hz}$ with a Novocontrol System by means of two different equipments. A Solartron-Schlumberger gain/phase analyzer SI1260 supplemented with a broad dielectric converter allowed the dielectric relaxation to be measured in the range $10^{-2} \mathrm{~Hz}$ up to $10^{7} \mathrm{~Hz}$. For higher frequencies $\left(10^{6} \mathrm{~Hz}-10^{9} \mathrm{~Hz}\right)$, a Hewlett-Packard impedance analyzer HP4191A based on the principle of a reflectometer was used. The error in the determination of the loss tangent of the dielectric constant was around $10^{-4}$ for the first set up and $10^{-2}$ for the second one. Overlapping of the spectra at the same temperature was possible by means of the common decade of both equipments and small corrections were motivated by the uncertainties in the determination of the sample thickness. Measurements were made under isothermal conditions each $5 \mathrm{~K}$ covering the temperature range $195 \mathrm{~K} \leqslant T$ $\leqslant 435 \mathrm{~K}$ with temperature stability better than $0.1 \mathrm{~K}$. 
Sample holders, that act as electrodes, were parallelplate capacitors of $20 \mathrm{~mm}$ diameter for the low frequency setup and $5 \mathrm{~mm}$ diameter for the high frequency one. The thickness of the sample was $0.1 \mathrm{~mm}$.

\section{Neutron scattering}

\section{Neutron spin echo}

The basis of this technique is founded in coding the neutron velocity change in the scattering process for each neutron individually into its spin rotation. ${ }^{22}$ The Larmor precession of the neutron spin in an external magnetic field uses the neutron spin as an internal clock. Starting with a well defined neutron spin state, the application of magnetic precession fields before and after the scattering event results in a polarization of the neutron that depends only on the velocity difference of each neutron individually to first order irrespective of its initial velocity. Energy resolution and monochromatization of the incident beam are thus decoupled, and resolutions in energy of $\delta E / E \approx 10^{-5}$ can be achieved with an incident neutron spectrum of $20 \%$ bandwidth.

The final polarization of the neutron beam in a NSE experiment is directly related to the normalized intermediate scattering function $S(Q, t) / S(Q) .{ }^{22}$ The time $t$ depends on the wavelength $\lambda$ of the neutron, the precession field $B$ and the length $L_{B}$ of the precession field, $t \propto B L_{B} \lambda^{3}$. Thus the time evolution of the structure factor can be followed varying $B$. Our experiment was performed by means of the spectrometer IN11 at the Institute Laue-Langevin (ILL) in Grenoble, France. Since due to the spin flip scattering the incoherent scattering contributions are strongly suppressed, the signal from the deuterated PVCd sample is nearly entirely due to coherent scattering revealing the pair correlation function.

By using an incident wavelength of $\lambda=4.3 \AA$, six temperatures in the range $390 \mathrm{~K} \leqslant T \leqslant 460 \mathrm{~K}$ were investigated at the first maximum of the static structure factor peak $Q_{\max }$ $=1.2 \AA^{-1} .{ }^{23}$ The Fourier time accessible was $3 \mathrm{ps} \leqslant t$ $\leqslant 1.8 \mathrm{~ns}$. The thickness of the sample was $2 \mathrm{~mm}$ and typical measuring times were around 3-4 h. The instrumental resolution function was measured from the elastic scattering of the sample at $4 \mathrm{~K}$. The instrumental background from the cryostat as well as from the $\mathrm{Al}$ container were measured separately and subtracted from the experimental spectra using the appropriate transmission factors. The background corrected spectra were divided by the resolution function in order to obtain the normalized intermediate dynamic structure factor $S(Q, t) / S(Q)$.

\section{Backscattering}

The incoherent scattering function (corresponding to the self-correlation function of the hydrogen atoms) was studied on the protonated sample by means of the neutron backscattering (BS) spectrometer IN10 also at the ILL. The incident wavelength was $\lambda=6.28 \AA$, providing an energy resolution of $\delta E \approx 1.2 \mu \mathrm{eV}$ [full width at half maximum (FWHM)]. Due to experimental problems in the smallest angles detectors, the $Q$-range covered was $0.4 \leqslant Q \leqslant 2 \AA^{-1}$. The sample (thickness: $0.17 \mathrm{~mm}$ ) was filled in a flat $\mathrm{Al}$ con- tainer yielding a transmission of about $92 \%$. The measurements were performed in a temperature range $380 \mathrm{~K} \leqslant T$ $\leqslant 430 \mathrm{~K}$. Typical measuring times at each temperature were $12 \mathrm{~h}$. The instrumental resolution function was determined from the scattering of the sample at $2 \mathrm{~K}$. Initial data treatment was carried out correcting for effects of detector efficiencies, scattering from sample container, and instrumental background. Finally at each temperature the incoherent structure factor $S_{\text {inc }}(Q, \omega)$ was obtained as a function of the energy change during scattering $\hbar \omega$ and the change of wave vector $Q$.

\section{Small angle neutron scattering}

The measurements of the chain form factor and the static structure factor $S(Q)$ in the low $Q$-range were carried out by means of the small angle neutron scattering (SANS) diffractometer KWS1 at the FRJ-2 research reactor in Jülich, Germany. The sample (thickness: $1 \mathrm{~mm}$ ) was in between quartz plates of $2 \mathrm{~mm}$, and the brass sample holder was placed in an electrically heated furnace. The sample aperture was 5 $\times 5 \mathrm{~mm}^{2}$. An incident wavelength of $\lambda=7 \AA$ with a wavelength spread $\delta \lambda / \lambda=0.2$ was used. Performing measurements at two detector positions (sample-detector distances of $1.25 \mathrm{~m}$ and $4 \mathrm{~m}$ ), a $Q$-range $0.01 \AA^{-1} \leqslant Q \leqslant 0.25 \AA^{-1}$ was covered. The raw data were corrected for empty cell and hall background scattering. Absolute scattering intensities were obtained in relating the scattered intensity to the known incoherent scattering level of a secondary polyethylene standard,

$$
\frac{\partial \Sigma}{\partial \Omega}(Q)=\frac{(x T)_{s}}{x T}\left(\frac{D}{D_{s}}\right)^{2} \frac{S(Q)}{S_{s}(Q)}\left(\frac{\partial \Sigma}{\partial Q}\right)_{S}(Q),
$$

where $s$ stands for the standard and $x, T$, and $D$ are thickness, transmission, and detector distance.

The static structure factor was determined from the scattering of a fully deuterated PVCd sample. The chain form factor was obtained from measurements on blends of $\mathrm{PVCd} /$ PVCh with two different concentrations $(2 \%$ and $10 \%$ of deuterated chains in protonated matrices). We investigated several temperatures in the range $360 \mathrm{~K} \leqslant T \leqslant 470 \mathrm{~K}$ (five temperatures for both blend samples and in addition $450 \mathrm{~K}$ for PVCd). After appropriate equilibration, a measuring time of around $30 \mathrm{~min}$ was employed at each temperature. The sample transmission was measured for all temperatures. After correcting the data pixel by pixel, a radial average was performed.

\section{EXPERIMENTAL RESULTS}

\section{A. Small angle neutron scattering}

SANS techniques access scattering phenomena corresponding to typical lengths of 10-1000 . These scales may relate to inhomogeneities of very different origins, such as the presence of different phases in the material, density fluctuations, clusters, sizes of chains,.... Figure 1(a) shows the SANS results obtained for pure PVCd for temperatures close and above $T_{g}$. All curves display similar features: (i) the presence of a broad peak, the intensity and maximum position of which vary with $T$ and (ii) a strong intensity increase 


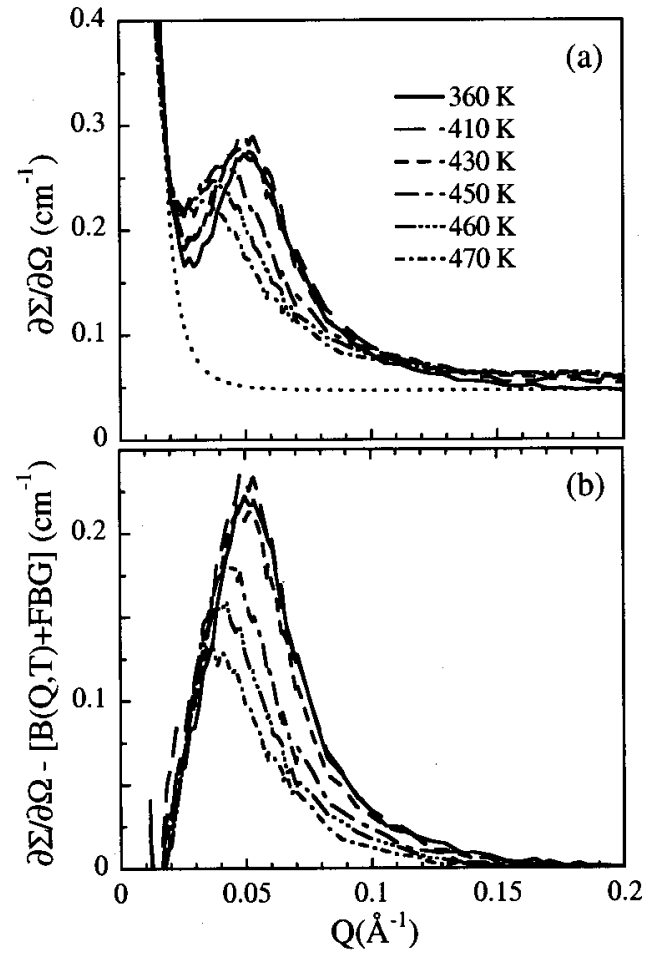

FIG. 1. (a) Small angle neutron scattering results on PVCd for the different temperatures indicated. As an example for $360 \mathrm{~K}$, the dotted line shows the additional contributions to the peak $B(Q, T)+\mathrm{FBG}$ (see the text). (b) Results from subtracting the additional contributions from the experimental data at the same temperatures as in (a).

at very low $Q$-values (below $0.02 \AA^{-1}$ ). This last feature relates most probably to the existence of bubbles or some residual porosity in the material originating from the sample preparation procedure. The scattering contrast for such holes is especially high for a deuterated material. The correlation peak is the salient feature in these diffraction curves. It evidences the existence of a long period within the PVCd sample reflecting some periodic modulation of the density. These results corroborate earlier findings on PVC by small angle x-ray scattering (SAXS) (Refs. 3-6, 8, 9) and SANS. ${ }^{5,7}$

In order to characterize the correlation peaks in the simplest way, the contribution at very low $Q$-values, $B(Q, T)$, may be subtracted. Furthermore, we subtract a flat background FBG. Its value is determined from the high $Q$-limit of the intensity. The low $Q$ scattering $B(Q, T)$ was described by a power law $B(Q, T)=C Q^{-a}$, where the prefactor $C$ and the exponent $a$ were found to be slightly $T$-dependent. The value $a \sim 4$ is characteristic for Porod-scattering from objects larger than the accessible length scales. As an example, the dotted line in Fig. 1(a) represents the total contribution $B(Q, T)+$ FBG to be subtracted from the $360 \mathrm{~K}$ curve. For all the temperatures investigated Fig. 1(b) displays the remaining peaks after the proper subtraction of these additional contributions. For $T>430 \mathrm{~K}$ the maximum position and the intensity of these peaks are strongly $T$-dependent. This is evident in Fig. 2. The thermal evolution of the correlation length $L=2 \pi / Q_{\max }^{\text {SANS }}\left(Q_{\max }^{\mathrm{SANS}}\right.$, maximum position) and the integrated peak intensity are shown in Figs. 2(a) and 2(c),

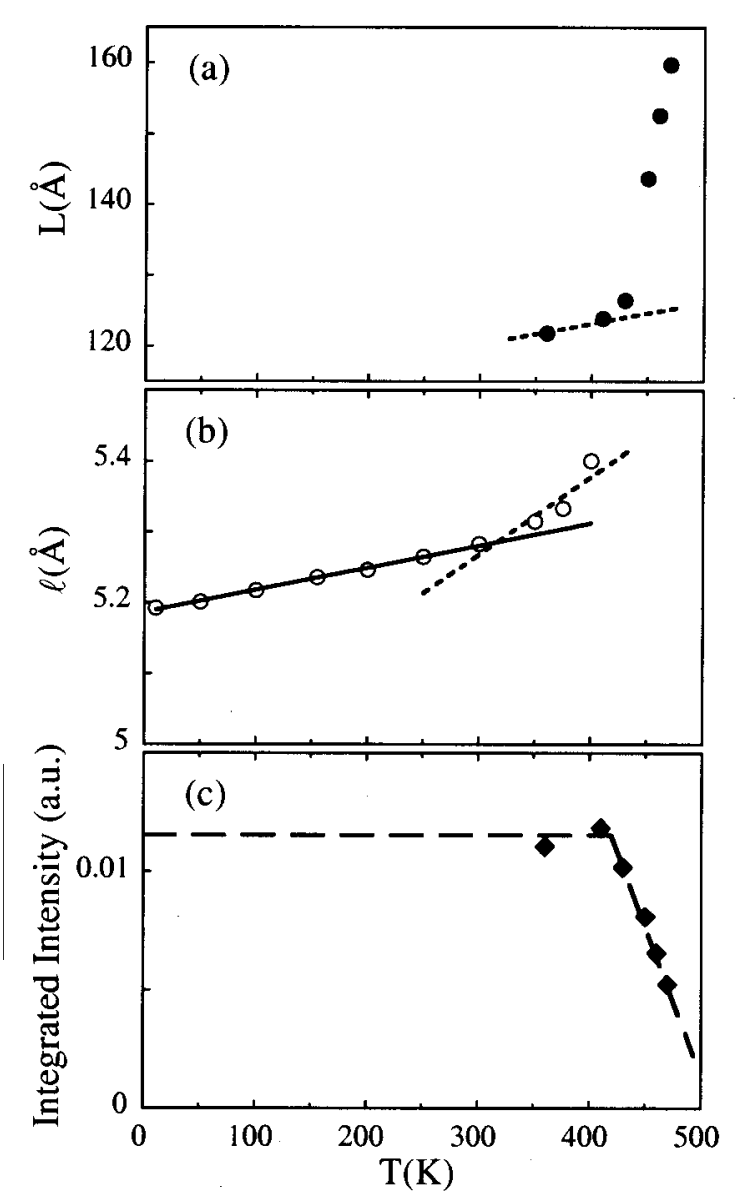

FIG. 2. Temperature dependence of (a) the characteristic length associated to the SANS peak $L$, (b) the characteristic length associated to the first maximum of the static structure peak $\ell$, and (c) the integrated intensity of the SANS peak (additional contributions previously subtracted). The solid and dotted lines show the $T$-dependence obtained from the expansion coefficient in the glassy and supercooled liquid states, respectively. Dashed lines in (c) are guides for the eye.

respectively. For comparison, another correlation length, namely, that associated to the main ("first") static structure factor peak defined as $\ell=2 \pi / Q_{\max }$ is depicted in Fig. 2(b) (see Discussion). An important change in the slope of $L(T)$ takes place in the temperature range 430-450 K. Also the intensity shows a strong decrease with increasing $T$ that starts in the same $T$-range.

In addition to the SANS structure factor of the PVCd sample, also the chain form factor within the amorphous matrix was studied. This is possible only with neutron scattering techniques, due to the different neutron scattering lengths of deuterium and hydrogen nuclei. For that purpose, the SANS signals from two dilute blends with PVCd concentrations $\phi_{\mathrm{D}}=2 \%$ and $\phi_{\mathrm{D}}=10 \%$ in PVCh were investigated in the same $Q$ - and temperature-range. Figure 3 depicts the measured cross sections for the $\phi_{\mathrm{D}}=10 \%$ sample at three different temperatures and for the two concentrations at $430 \mathrm{~K}$ in its insert. The small incoherent backgrounds, determined from the high $Q$-limit of the intensities, have already been subtracted from these data. If the isotopic interaction between PVCh and PVCd chains is negligible (i.e., FloryHuggins parameter $\chi=0$ ), the scattering from the blend is given as 


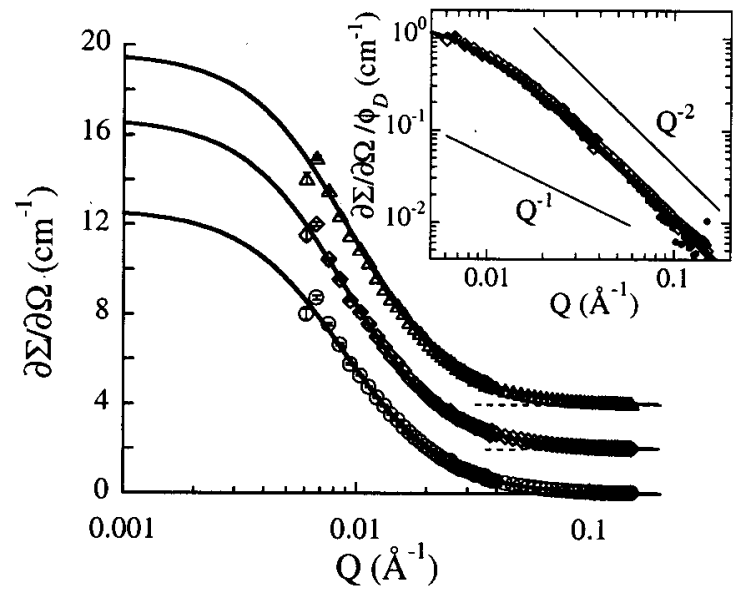

FIG. 3. Momentum transfer dependence of the cross sections measured by SANS for the $10 \% \mathrm{PVCd} / \mathrm{PVCh}$ blend at $360 \mathrm{~K}(\bigcirc), 430 \mathrm{~K}(\diamond)$ and $460 \mathrm{~K}$ $(\triangle)$. The $430 \mathrm{~K}$ and $460 \mathrm{~K}$ data have been shifted for clarity. The dotted horizontal lines show their corresponding new origins. Insert: cross sections divided by the concentration for the $10 \%(\diamond)$ and $2 \%(\bullet)$ samples at $430 \mathrm{~K}$. Straight lines indicate $Q^{-2}$ and $Q^{-1}$ slopes. The solid lines though the points both in the figure and in the insert are the descriptions obtained in terms of Eqs. (2)-(4).

$$
\begin{aligned}
\frac{\partial \Sigma}{\partial \Omega}(Q)= & \phi_{\mathrm{D}}\left(1-\phi_{\mathrm{D}}\right) \frac{\left(\rho_{\mathrm{H}}-\rho_{\mathrm{D}}\right)^{2}}{d} \\
& \times \int_{0}^{\infty} M W(M) P_{\text {chain }}(Q, M) d M,
\end{aligned}
$$

where $\rho_{\mathrm{H}}$ and $\rho_{\mathrm{D}}$ stand for the scattering length densities of the protonated and deuterated samples respectively. $P_{\text {chain }}(Q, M)$ is the chain form factor. For Gaussian chains, $P_{\text {chain }}(Q, M)$ is expressed by the well known Debye scattering function,

$$
P_{\text {chain }}(Q, M)=\frac{2}{Q^{4} R_{g}^{2}}\left[\exp \left(-Q^{2} R_{g}^{2}\right)-1+Q^{2} R_{g}^{2}\right]
$$

where the radius of gyration $R_{g}$ implicitly depends on $M$ as $R_{g} \propto M^{0.5} . W(M)$ provides the correction for the polydispersity. A Schultz-Zimm-Flory distribution,

$$
W(M)=\frac{b^{h+1}}{\Gamma(h+1)} M^{h} \exp (-b M)
$$

with $b=(h+1) / M_{w}$ and $h=\left(\left(M_{w} / M_{n}\right)-1\right)^{-1}$ was assumed.

Due to the low crystallinity of the sample (see Discussion), the contrast factor $\left(\rho_{\mathrm{H}}-\rho_{\mathrm{D}}\right)^{2}$ was calculated on the basis of amorphous densities only. With the polydispersity index $M_{w} / M_{n}=2$ (see Experiment), in the fitting procedure only two parameters were varied: the average radius of gyration and the average molecular weight. The fits of the experimental data in terms of Eqs. (2)-(4) are shown in Fig. 3 as solid lines. The agreement is highly satisfactory. The wide $Q$-range accessed allows to accurately determine the slope of $\partial \Sigma / \partial \Omega(Q)$ at high $Q$. There we find $\partial \Sigma / \partial \Omega(Q) \propto Q^{-2}$, underlining the Gaussian chain conformation.

The values obtained for the fitting parameters from the $\phi_{\mathrm{D}}=10 \%$ data are listed in Table I for the different temperatures investigated. The weight-average $M_{w}$ can be deduced from the cross section at $Q=0\left[P_{\text {chain }}(Q=0)=1\right]$. From $\partial \Sigma / \partial \Omega(Q=0)$, we derive $M_{w} \approx(75 \pm 9) \times 10^{3} \mathrm{~g} / \mathrm{mol}$. The error is mainly determined by the temperature dependence of the density and the accuracy of the in situ measurement of the transmission in the experiment $(\approx 5 \%)$. We note that the corresponding value for $M_{n} \approx 37.5 \times 10^{3} \mathrm{~g} / \mathrm{mol}$ is very close to that obtained for this sample by other characterization techniques (see Experiment). This consistency provides additional support to the description of the form factors in terms of Gaussian chains. At $430 \mathrm{~K}$, from the measurements from the $\phi_{\mathrm{D}}=10 \%$ sample a $w$-average radius of gyration $R_{g_{w}}=92 \pm 1 \AA$ is obtained; for the $\phi_{\mathrm{D}}=2 \%$ blend, the resulting value is $R_{g_{w}}=105 \pm 4 \AA$. The random-walk statistics is reflected in the relation $R_{g} \sim M^{0.5}$ implicitly assumed in the fitting procedure. We find that $R_{g}=(0.34 \pm 0.01) M^{0.5}$. Beside some uncertainties due to the reduced coherent scattering, both blends $\left(\phi_{\mathrm{D}}=2 \%\right.$ and $\left.10 \%\right)$ give very similar results. This may be seen from the insert in Fig. 3, where at $430 \mathrm{~K}$ results for both concentrations are displayed. With the $\phi_{\mathrm{D}}=2 \%$ sample slightly higher values are deduced for $M_{w}$ (5\% to $10 \%$ difference). These differences may be due to inaccurate volume fractions and worse signal to noise ratios in the $\phi_{\mathrm{D}}=2 \%$ sample.

\section{B. Dielectric spectroscopy}

Figure 4 presents representative data for the imaginary part of the dielectric constant $\varepsilon^{\prime \prime}(\omega)$ for PVC at different temperatures. As can be appreciated in Fig. 4(a), below $T_{g}$

TABLE I. Parameters characterizing the structural properties of the PVC sample investigated. The radius of gyration $R_{g_{z}}$ and the $w$-averaged molecular weight

\begin{tabular}{|c|c|c|c|c|c|c|c|}
\hline$T(\mathrm{~K})$ & $R_{g_{z}}(\AA)$ & $\begin{array}{c}M_{w} \times 10^{-3} \\
(\mathrm{~g} / \mathrm{mol})\end{array}$ & $\phi_{c}$ & $R_{m}(\AA)$ & $\eta$ & $2 R_{\mathrm{HS}}(\AA)$ & $L(\AA)$ \\
\hline 360 & $113 \pm 2$ & $68 \pm 3$ & $0.041 \pm 0.002$ & $30.5 \pm 0.3$ & 0.23 & $109 \pm 2$ & $118 \pm 10$ \\
\hline 410 & $113 \pm 1$ & $75 \pm 3$ & $0.041 \pm 0.001$ & $31.6 \pm 0.1$ & 0.22 & $110 \pm 2$ & $122 \pm 10$ \\
\hline 430 & $114 \pm 1$ & $81 \pm 4$ & $0.038 \pm 0.001$ & $31.6 \pm 0.1$ & 0.22 & $114 \pm 2$ & $122 \pm 10$ \\
\hline 450 & $\ldots$ & $\ldots$ & $0.024 \pm 0.001$ & $32.2 \pm 0.2$ & 0.20 & $130 \pm 3$ & $148 \pm 10$ \\
\hline 460 & $108 \pm 2$ & $88 \pm 4$ & $0.019 \pm 0.001$ & $32.3 \pm 0.2$ & 0.20 & $141 \pm 3$ & $158 \pm 10$ \\
\hline 470 & $92 \pm 1$ & $66 \pm 3$ & $0.016 \pm 0.001$ & $32.5 \pm 0.3$ & 0.18 & $146 \pm 4$ & $170 \pm 10$ \\
\hline
\end{tabular}
$M_{w}$ have been obtained from the SANS study of the $10 \%$ PVCd/PVCh sample. The description of the heterogeneous structure shown by the PVCd sample in terms of the Percus-Yevick approach (see text) yields the values for crystallinity $\phi_{c}$, the average radius of the microcrystallite $R_{m}$, the effective interaction radius $R_{\mathrm{HS}}$ and hard sphere volume fraction $\eta$ here listed. The correlation length $L$ is that determined from the SANS peak position. 


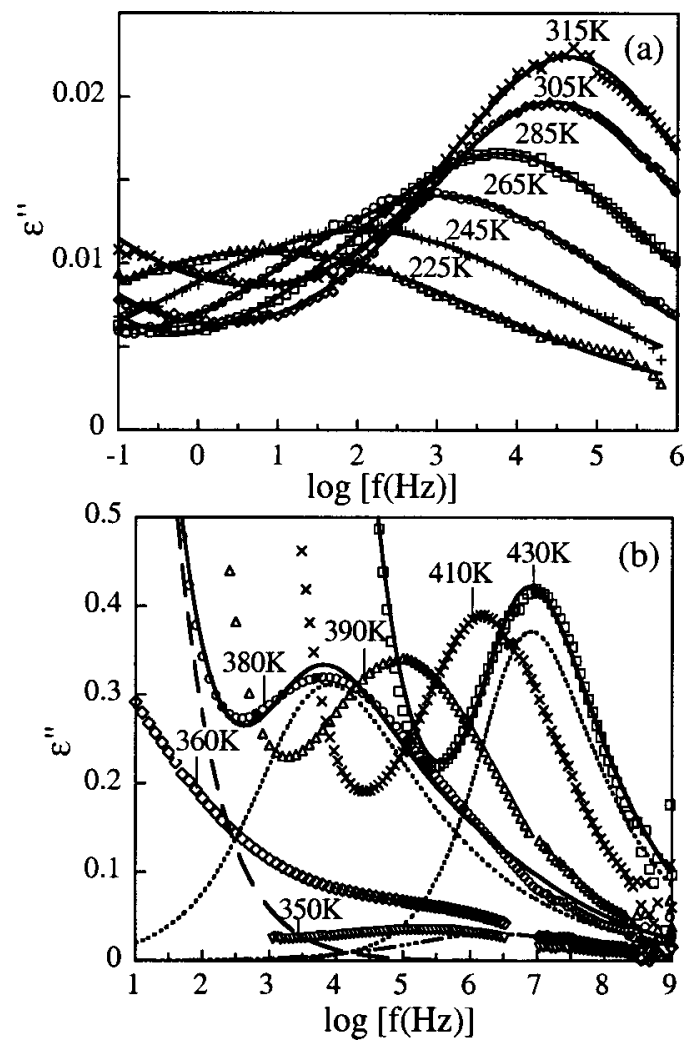

FIG. 4. Frequency dependence of the dielectric losses of PVC in the sub- $T_{g}$ region (a) and close and above $T_{g}$ (b) at the temperatures indicated. Symbols correspond to experimental data; solid lines represent the fitting curves resulting from the standard description. They give account mainly for the $\beta$-relaxation in (a) and include the three contributions of Eq. (5) for $380 \mathrm{~K}$ and $430 \mathrm{~K}$ in (b). For $380 \mathrm{~K}$ the individual contributions are shown: the conductivity (dashed line), the $\beta$-relaxation (dashed-dotted line) and the $\alpha$-relaxation described in terms of a single KWW (dotted line). This last contribution is depicted also by a dotted line for $430 \mathrm{~K}$.

the secondary relaxation is the main responsible of the dielectric loss features. As the temperature increases approaching $T_{g}$, the $\alpha$-relaxation starts to be observed in the frequency window [see, e.g., the curve corresponding to $360 \mathrm{~K}$ in Fig. 4(b)]. Above $T_{g}$ with increasing temperature the two loss peaks corresponding to the $\alpha$ and $\beta$ processes approach each other, the $\alpha$-relaxation taking place at lower frequencies than the $\beta$-relaxation. At high temperatures (e.g., $430 \mathrm{~K}$ ) the relaxations merge and only one peak can be resolved in the experimental dielectric window. On the other hand, the presence of an additional third contribution at low frequencies and high temperatures is evident. This contribution, that increases with $T$, can be attributed to the conductivity of the sample and also to interfacial polarization processes.

Taking into account these three identified contributions, the total permittivity $\varepsilon^{*}(\omega)$ can be written as

$$
\varepsilon^{*}(\omega)=\varepsilon_{c}^{*}(\omega)+\varepsilon_{\beta}^{*}(\omega)+\varepsilon_{\alpha}^{*}(\omega),
$$

where $c, \beta$, and $\alpha$ stand for the conductivity, $\beta$ - and $\alpha$-process, respectively. In the following, the expressions for the permittivity contributions of these processes in conventional glassforming polymers are given.
If the low frequency contribution were purely dissipative, $\varepsilon_{c}^{\prime \prime}(\omega)$ would be inversely proportional to the frequency, i.e.,

$$
\varepsilon_{c}^{\prime \prime}(\omega)=\frac{\sigma_{c}}{\omega^{\kappa}}
$$

with

$$
\kappa=1,
$$

where $\sigma_{c}$ strongly depends on temperature. Values of $\kappa$ lower than 1 in Eq. (6a) would reflect the contribution of interfacial processes.

Concerning the relaxation processes, the permittivity $\varepsilon_{\alpha, \beta}^{*}(\omega)$ can be expressed in terms of the response function of the corresponding process in the frequency domain $\Phi_{\alpha, \beta}^{*}(\omega)$, that is related to the relaxation function in the time domain $\varphi_{\alpha, \beta}(t)$ via Laplace transformation,

$$
\begin{aligned}
\frac{\varepsilon_{\alpha, \beta}^{*}(\omega)-\varepsilon_{\infty_{\alpha, \beta}}}{\Delta \varepsilon_{\alpha, \beta}} & =\Phi_{\alpha, \beta}^{*}(\omega) \\
& =\int_{0}^{\infty}-\frac{\partial \varphi_{\alpha, \beta}(t)}{\partial t} e^{-i \omega t} d t .
\end{aligned}
$$

Here $\Delta \varepsilon_{\alpha, \beta}$ and $\varepsilon_{\infty, \beta}$ are the dielectric strength and the high frequency limit of the real part of the permittivity value, respectively.

It is well established that in polymers $\varphi_{\alpha}(t)$ and $\varphi_{\beta}(t)$ are not simple exponentials, or, equivalently, $\Phi_{\alpha}^{*}(\omega)$ and $\Phi_{\beta}^{*}(\omega)$ are not simple Debye functions. As can be deduced from Fig. 4, in the case of PVC this is also evident (the FWHM of a Debye peak is 1.14 decades).

In the case of the $\beta$-relaxation, the dielectric function of polymers is commonly described in terms of a superposition of Debye elemental processes. These are supposed to be thermally activated, i.e., the characteristic time for a given elemental process $\tau_{\beta}$ is given by

$$
\tau_{\beta}(E)=\tau_{\beta}^{o} \exp \left(\frac{E}{k_{B} T}\right)
$$

( $\tau_{\beta}^{o}, T$-independent prefactor; $k_{B}$, Boltzmann constant). Due to the disorder in the system, the values for the energy barrier $E$ are distributed according to a distribution function $g_{\beta}(E)$. With this, for the permittivity we arrive at

$$
\frac{\varepsilon_{\beta}^{*}(\omega)-\varepsilon_{\infty_{\beta}}}{\Delta \varepsilon_{\beta}}=\int_{0}^{\infty} g_{\beta}(E) \frac{1}{1+i \omega \tau_{\beta}(E)} d E .
$$

A Gaussian distribution of energy barriers $g_{\beta}(E)$,

$$
g_{\beta}(E)=\frac{1}{\sigma_{E} \sqrt{2 \pi}} \exp \left[-\frac{1}{2}\left(\frac{E-E_{o}}{\sigma_{E}}\right)^{2}\right]
$$

of variance $\sigma_{E}^{2}$ and average value $E_{o}$ usually gives a good description of the experimental data at $T<T_{g}$.

In glassforming polymers the relaxation function for the $\alpha$-relaxation in general can be well described by means of the Kohlrausch-Williams-Watts (KWW) or stretched exponential function, 


$$
\varphi_{\alpha}(t)=\exp \left[-\left(\frac{t}{\tau_{w}}\right)^{\beta}\right],
$$

where $\tau_{w}$ is the characteristic time of the relaxation and $\beta$ is the shape parameter, $0<\beta<1$, giving account for the nonexponential behavior. For most of the polymers the value of $\beta$ is found to be hardly dependent on $T$ and to be in the range $0.4-0.5$. As dielectric measurements are usually performed in the frequency domain, an analytical expression for the response function $\Phi_{\alpha}^{*}(\omega)$ facilitates the data treatment. However, the Laplace transform of KWW functions in the general case does not have an analytical form. It was shown nevertheless that it can be well described by Havriliak Negami (HN) functions, if the shape parameters fulfill certain relationships. ${ }^{24,25}$ Therefore, for expressing $\Phi_{\alpha}^{*}(\omega)$ we made use of HN-functions,

$$
\Phi_{\alpha}^{*}(\omega)=\Phi_{\mathrm{HN}}^{*}(\omega)=\frac{1}{\left[1+\left(i \omega \tau_{\mathrm{HN}}\right)^{\alpha}\right]^{\gamma}},
$$

where $\alpha$ and $\gamma$ are the shape parameters and $\tau_{\mathrm{HN}}$ is the characteristic time. We restricted ourselves to the $\mathrm{HN}$-family functions which describe well the Laplace transform of KWW functions. This is achieved by the imposing the relation,

$$
\gamma=1-0.812(1-\alpha)^{0.387} .
$$

Under these circumstances the relationships between HNand KWW-shape parameters and their characteristic times are

$$
\begin{aligned}
& \alpha \gamma=\beta^{1.23}, \\
& \log \left[\frac{\tau_{\mathrm{HN}}}{\tau_{w}}\right]=2.6(1-\beta)^{0.5} \exp (-3 \beta) .
\end{aligned}
$$

Since we are dealing with only one independent shape parameter, the results will be given in terms of the parameter $\beta$.

For conventional glassforming polymers the dielectric spectra can be described by means of Eq. (5) and considering the above given expressions for the different contributions. Due to the high number of parameters involved, the first step in the usual procedure consists of characterizing the secondary relaxation in the temperature range below $T_{g}$. There, the conductivity does not appear and the $\alpha$-relaxation contribution is not yet present or is still weak. Thereafter, under the assumption that the parametrization obtained for the $\beta$-process can be extrapolated to higher $T$ above $T_{g}$, the fit of the experimental data allows to get the information on the structural relaxation and the conductivity. This approximation is found to be valid in the temperature range where the $\alpha$ - and $\beta$-processes are separated. ${ }^{16}$

Figure 4(a) shows that the description of the loss peaks of PVC in terms of this scenario for the $\beta$-process is very good for $T<T_{g}$. We note that a power law accounting for the small contribution of the high frequency tail of the $\alpha$-process was considered in these descriptions for $T$ $>255 \mathrm{~K}$. Thus the parameters characterizing the secondary processes $\left(\tau_{\beta}^{o}, \sigma_{E}, E_{o}\right.$, and the dielectric strength $\left.\Delta \varepsilon_{\beta}\right)$ can be well determined in this sub- $T_{g}$ region. The results are shown in Fig. 5. In the upper part of the figure the charac-
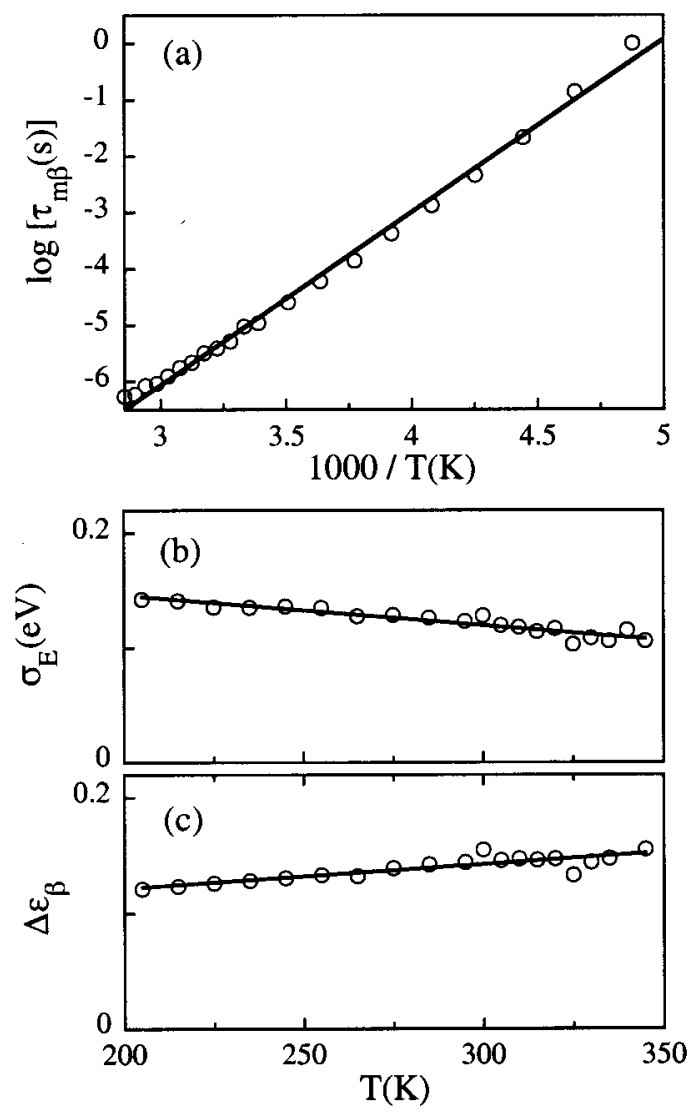

FIG. 5. Temperature dependence of the parameters characterizing the dielectric $\beta$-relaxation in PVC: (a) characteristic time corresponding to the maximum of the losses; (b) width of the distribution function of energy barriers; (c) relaxation strength. Solid lines correspond to (a) an Arrheniustype temperature dependence; (b) and (c) linear regression fits as function of the temperature.

teristic time of the $\beta$-relaxation $\tau_{m \beta}$ is represented. It is obtained from the maximum of the loss peak and corresponds to the relaxation time for the most probable value of the energy barrier, $\tau_{m \beta}=\tau_{\beta}\left(E_{o}\right)$. From the fit of these data to an Arrhenius law, values of $0.61 \mathrm{eV}$ for $E_{o}$ and $5.5 \times 10^{-16} \mathrm{~s}$ for $\tau_{\beta}^{o}$ are obtained. Concerning the width of the energy barrier distribution function $g_{\beta}(E)$, a slight decrease of $\sigma_{E}$ with increasing temperature is found, $\sigma_{E}[\mathrm{eV}]=0.198-2.6$ $\times 10^{-4} T[\mathrm{~K}]$ [see Fig. 5(b)]. The strength $\Delta \varepsilon_{\beta}$ follows approximately the linear $T$-dependence given by $\Delta \varepsilon_{\beta}=0.08$ $+2.1 \times 10^{-4} T[\mathrm{~K}]$ that is shown in Fig. 5(c) by the solid line.

Above $T_{g}$ the three contributions of Eq. (5) are present in the dielectric spectra. The loss peak corresponding to the $\beta$-process was extrapolated from $T<T_{g}$. Close to $T_{g}$, the description obtained for the experimental spectra was quite unsatisfactory, as can be appreciated from the fitting curves shown in Fig. 4(b) as examples. Even allowing the exponent $\kappa$ in Eq. (6a) to take values smaller than 1, the shape of the experimental data cannot be well described when the functional form of the $\alpha$-contribution is restricted to correspond to a KWW function. The best description of the data using a $T$-independent $\kappa$ value $(\kappa=0.67)$, gave the results shown in Fig. 6 for the parameters characterizing the $\alpha$-relaxation. The shape parameter $\beta$ is found to depend strongly on tempera- 


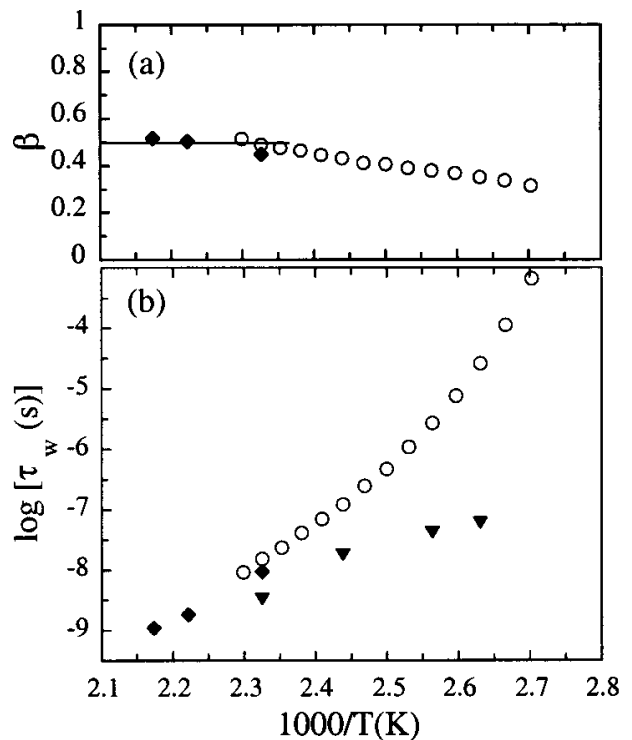

FIG. 6. Temperature dependence of the parameters characterizing the $\alpha$-relaxation in PVC when described by a single KWW function: (a) Shape parameter; (b) Relaxation time. Dielectric spectroscopy: $\bigcirc$; coherent scattering at $Q_{\max }=1.2 \AA^{-1}$ (NSE): $\bullet$; incoherent scattering at the same $Q$-value (BS): $\boldsymbol{\nabla}$. Solid horizontal line in (a) indicates the asymptotic value of $\beta$ at high temperatures.

ture [see Fig. 6(a)], increasing from $\approx 0.3$ at $T_{g}$ up to $\approx 0.5$ at the highest $T$ investigated. This indicates a pronounced narrowing of the response function. As we have mentioned before, such behavior is anomalous for glassforming polymers. On the other hand, as can be seen in Fig. 6(b), the characteristic times show the typical non-Arrhenius behavior of glassforming systems.

\section{Neutron spin echo: Coherent scattering at the first static structure factor peak}

The dynamic structure factor obtained from the NSE measurements on PVCd at the first structure factor peak $Q_{\max }\left(Q_{\max }=1.2 \AA^{-1}\right)$ and different temperatures above $T_{g}$ is represented in Fig. 7. At first sight, two features can be deduced: (i) the curves display a clearly stretched time evolution, and (ii) the characteristic time of the decay becomes faster with increasing temperature.

As we have already pointed out, the decay of the correlations of the density fluctuations in the neighborhood of $Q_{\max }$ is governed by the $\alpha$-relaxation. The functional form characteristic for this process is a KWW function. In order to account for the decay of correlations related to processes taking place at faster times than those accessed by IN11, e.g., vibrations, the so-called "fast dynamics," etc., a prefactor (Debye-Waller factor) $A_{Q_{\max }}(T)$ has to be introduced. Thus the dynamic structure factor at $Q_{\max }$ can be written as

$$
\frac{S\left(Q_{\max }, t\right)}{S\left(Q_{\max }\right)}=A_{Q_{\max }}(T) \exp \left[-\left(\frac{t}{\tau_{w}^{Q_{\max }}}\right)^{\beta}\right] .
$$

As can be seen in Fig. 7 for the highest temperatures investigated (dashed lines), this functional form allows a good description of the experimental data. The characteristic time $\tau_{w}^{Q_{\max }}$ as well as the shape parameter $\beta$ can only be indepen-

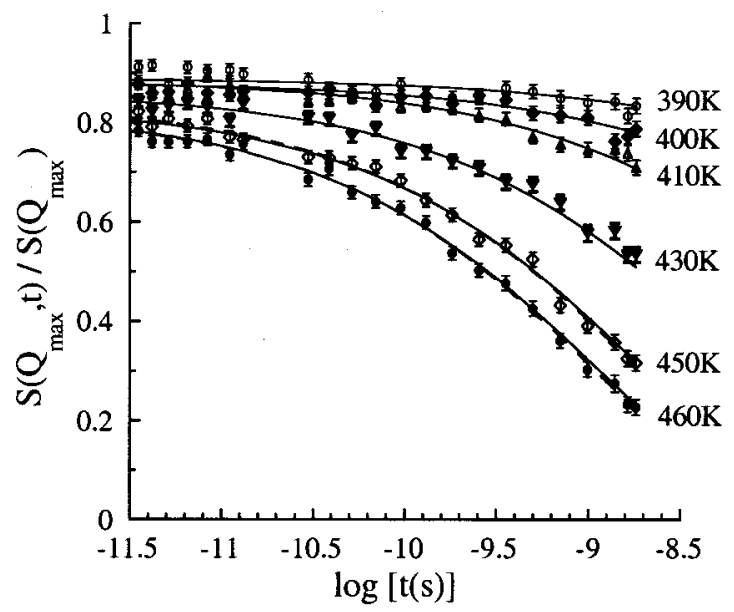

FIG. 7. Time evolution of the dynamic structure factor of PVC as measured by IN11 on a deuterated sample at the first static structure factor peak. The corresponding temperatures are indicated. Dashed lines are KWW [Eq. (15)] fitting curves for the two highest temperatures. Solid lines correspond to the fit results of the heterogeneous model proposed.

dently determined for $T \geqslant 430 \mathrm{~K}$, where an important portion of the decay takes place within the IN11 window. The results for $\beta$ and $\tau_{w}^{Q_{\max }}$ have been plotted in Fig. 6 for comparison with the dielectric results. Figure 6(a) shows that the values obtained for $\beta$ by both experimental techniques in the overlapping temperature are very much compatible. Towards high temperatures $\beta$ appears to increase more slowly than at lower temperatures, and a constant $\beta=0.5$ serves as a good approximation for $\beta$ at $T>430 \mathrm{~K}$. Furthermore in this temperature regime, the temperature dependence of $\tau_{w}^{Q_{\max }}$ agrees with the dielectric results.

\section{Backscattering: Incoherent scattering}

The scattering function measured by the backscattering spectrometer IN10 on PVCh is strongly dominated by the incoherent scattering contribution of the hydrogen atoms in the sample. In Fig. 8 some spectra corresponding to different $Q$-values and temperatures are compared with the instrumental resolution. In all these cases the spectra are broader than the resolution. The quasielastic broadening increases with increasing $Q$ and $T$.

Similar to the collective response at $Q_{\max }$, for a large number of polymer systems the results obtained in the $\alpha$-relaxation regime ${ }^{26-30}$ indicate that the incoherent scattering function $S_{\text {inc }}(Q, t)$ can be described by a KWW function. Furthermore a prefactor $A(Q, T)$ needs to be introduced, in order to account for fast processes and the Debye-Waller factor. Finally we have

$$
S_{\text {inc }}(Q, t)=A(Q, T) \exp \left[-\left(\frac{t}{\tau_{w}(Q, T)}\right)^{\beta}\right] .
$$

Most results for $\tau_{w}(Q, T)$ reported so far for glass forming polymers follow a power law in $Q$ which may be expressed by 


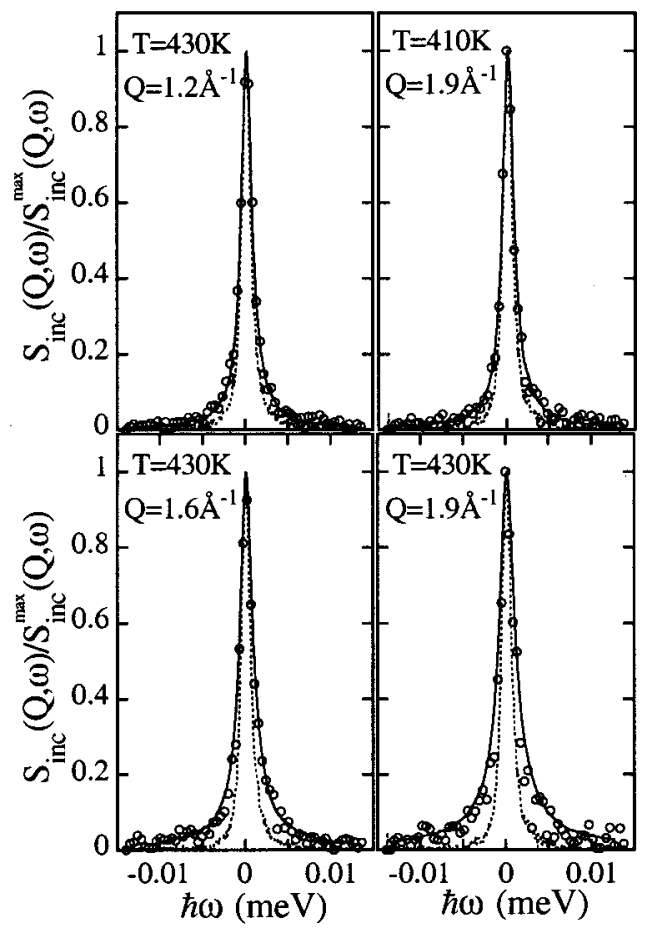

FIG. 8. Frequency dependence of the incoherent scattering function of PVC obtained by IN10 on a protonated sample normalized to its maximum value. The corresponding $T$ - and $Q$-values are indicated. Symbols correspond to experimental data. The instrumental resolution is also depicted (dotted line) for comparison. Solid lines show the description obtained by using the heterogeneous model.

$$
\tau_{w}(Q, T)=\tau_{0}(T)\left(\frac{Q}{Q_{0}}\right)^{-n}
$$

with $Q_{0}=1 \AA^{-1}$ to get the right time dimensions for the temperature dependent prefactor $\tau_{0}(T)$ and

$$
n=2 / \beta \text {. }
$$

This result reflects ${ }^{26}$ the Gaussianity of the scattering function, ${ }^{31}$ that can be written as

$$
\begin{aligned}
S_{\text {inc }}(Q, t) & =A(Q, T) \exp \left\{-\left[\frac{t}{\tau_{0}(T)}\right]^{\beta}\left(\frac{Q}{Q_{0}}\right)^{2}\right\} \\
& =A(Q, T) \exp \left[-\frac{\left\langle r^{2}(t)\right\rangle Q^{2}}{6}\right],
\end{aligned}
$$

where $\left\langle r^{2}(t)\right\rangle$ is the mean square displacement of the moving proton. Thus, the $\alpha$-relaxation corresponds to an anomalous diffusive process, where the mean square displacement increases sublinearly with time, $\left\langle r^{2}(t)\right\rangle=6 \mathcal{D} t^{\beta}$. Here, $\mathcal{D}$ is an "anomalous" diffusion coefficient determined by the $T$-dependent part of $\tau_{w}(Q, T), \mathcal{D} \approx \tau_{0}(T)^{-\beta}$. Let us now see what is found here for PVCh.

The experimental data-collected in the frequency domain, $S_{\text {inc }}(Q, \omega)$-were fitted to the result of convoluting the Fourier transform of Eq. (16) with the instrumental resolution. Following the procedure described in a previous work, ${ }^{29}$ multiple scattering corrections were also performed. These corrections were very small in our case, since (i) only results at large $Q$-values were available and (ii) the neutron

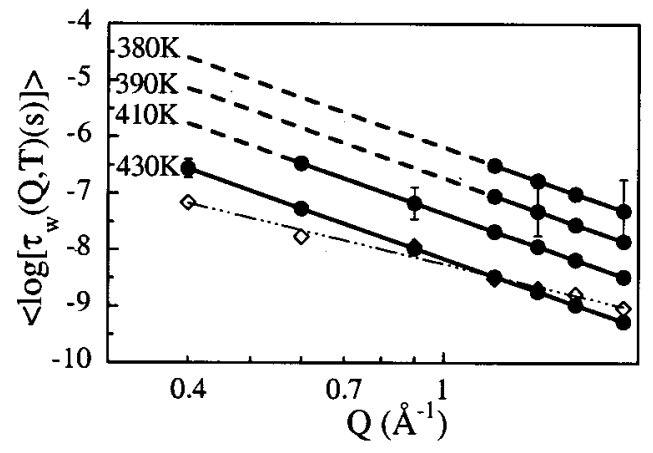

FIG. 9. Momentum transfer dependence of the characteristic times obtained from incoherent scattering at the temperatures indicated. Empty symbols correspond to the KWW times obtained from the fit of single detector spectra at $430 \mathrm{~K}$ to a single KWW with $\beta=0.5$. Dashed-dotted line is the result of the fit of these times to a power law with free exponent. Solid lines represent the power laws obtained from the fit of the experimental data by means of the heterogeneous model, the dashed line the corresponding extrapolation. The dots indicate the $Q$-values at which experimental spectra exist and show broadening with respect to the instrumental resolution. The bars show the FWHM of the distribution functions of KWW relaxation times used.

absorption of the $\mathrm{Cl}$ atoms is quite strong. In order to reduce the number of fit parameters, the value of the shape parameter $\beta$ was fixed to $\beta=0.5$ that was obtained from the coherent scattering results in the high temperature range. An acceptable description of the experimental spectra was achieved. The $Q$-dependent results for $\tau_{w}(Q, T)$ at $430 \mathrm{~K}$ are shown in Fig. 9 (empty symbols). Instead of the expected exponent value of $n=4$ an anomalously low value of $n$ $=2.7$ is found. Thus, the relation between $n$ and the stretching parameter $\beta$ strongly deviates from Eq. (17b). The characteristic relaxation times obtained for $Q=1.2 \AA^{-1}$ are depicted in Fig. 6(b) and compared with the results from the other techniques. As may be seen, the $T$-dependence of the times obtained from incoherent scattering is compatible with that of the dielectric times at the highest temperatures investigated, but strong deviations towards faster values are observed at $390 \mathrm{~K}$ and $380 \mathrm{~K}$.

\section{DISCUSSION}

\section{A. Structural heterogeneities}

The SANS results on the deuterated sample show clear evidence for the existence of a density modulation in the sample. In Fig. 2(a) we have displayed the temperature dependence of the corresponding correlation length $L$. $L$ was interpreted as the characteristic length scale of the density modulation in the sample, while the broadness of the correlation peak relates to the distribution of such distances. In Fig. 2 the results for the modulation length $L$ [Fig. 2(a)] are compared with the characteristic length $\ell$ obtained from the position of the first static structure factor peak, ${ }^{32} \ell$ $=2 \pi / Q_{\max }$ [Fig. 2(b)], that reflects the average interchain distance. We observe that both characteristic lengths exhibit a similar expansion for $T_{g}<T<430 \mathrm{~K}$. From the expansion coefficient in the supercooled liquid state $\alpha_{l} \approx 6.67$ $\times 10^{-4} \mathrm{~K}^{-1},{ }^{33}$ a linear expansion coefficient $\beta_{l}=\alpha_{l} / 3 \approx 2.2$ 
$\times 10^{-4} \mathrm{~K}^{-1}$ can be deduced. The dashed lines in Figs. 2(a) and 2(b) correspond to the laws $L / L_{0}=\ell / \ell_{0}=1+\beta_{l} T$. Thus the average domain size in this $T$-range simply follows thermal expansion. Furthermore, the intensity of the SANS peaks in this $T$-range is constant, indicating that the number of regions or the density contrast does not significantly change, i.e., the structural modulation remains unaltered. However, above $430 \mathrm{~K}$ a qualitatively different behavior sets on. The peak shifts very rapidly and the intensity strongly decreases with increasing $T$. This observation might relate to the melting of the "crystalline" parts in the sample, as it has been commonly interpreted by several authors. ${ }^{6,34}$

A detailed molecular description of the scattering pattern obtained in SANS, is difficult to realize. However, although details are lacking, a tentative interpretation of the heterogeneities could be provided if one simplifies considerably on the shape of the heterogeneity. The simplest approach then is to consider a system of polydisperse spherical particles, interacting by a hard-core potential in an amorphous matrix. The interparticle structure factor for such a system is liquidlike and well known for colloidal particles as the PercusYevick approach. We refer to the literature for the exact formulation. ${ }^{35}$ The intraparticle structure yields the average size of the inhomogeneity, i.e., of the microcrystallites and the distance between them. Their volume content $\phi_{c}$ or crystallinity degree in this case can be derived from the intensity, written in the decoupled approximation as

$$
\frac{\partial \Sigma}{\partial \Omega}(Q)=\phi_{c} V_{p}\left(\rho_{c}-\rho_{a}\right)^{2}\left\langle P_{c}(Q)\right\rangle_{z} I\left(Q, R_{m}, \eta, R_{H S}\right)+\mathrm{FBG} .
$$

Here, $\left\langle P_{c}(Q)\right\rangle_{z}$ is the $z$-averaged form-factor of a "crystalline" sphere of which a volume fraction $\phi_{c}$ is present. The size of such spheres is distributed according to a Gaussian function with variance $\sigma_{R_{m}}^{2}$. Knowing the temperature behavior of the mass density and the ratio of amorphous to crystalline density to be $1.37 / 1.53,{ }^{3}$ the temperature dependent scattering length densities $\rho_{c}(T)$ and $\rho_{a}(T)$ (subscripts $c, a$ denote crystalline, amorphous) can be calculated. The volume of the "crystallite" $V_{p}$ is determined by the mean radius $R_{m}, V_{p}=4 \pi R_{m}^{3} / 3 . \phi_{c}$ and $R_{m}$ are to be fitted for the form factor and $R_{\mathrm{HS}}$ and $\eta$, i.e., effective interaction radius and hard sphere volume fraction, for the structure factor. Whereas the crystallinity of PVC is hard to get, this model provides at least an estimate. The contrast factor was kept fixed at the appropriate temperature value. In addition, a flat incoherent background $\operatorname{FBG}(T)$ as discussed earlier was taken from the high- $Q$ limit. For three temperatures, Fig. 10 shows the description of the measured structure factor in terms of this model. The experimental data have been corrected for the low $Q$-increase as described above. The insert shows the separate contributions $I\left(Q, R_{m}, \eta, R_{\mathrm{HS}}\right)$ and $\left\langle P_{c}(Q)\right\rangle_{z}$ for $430 \mathrm{~K}$. The resulting fitting parameters are summarized in Table I. The average size of the microcrystallites is steady around $32 \AA$ radius $\left(\sigma_{R_{m}}^{2} \approx 0.1-0.15\right)$. In contrast, above $430 \mathrm{~K}$ the effective hard-sphere interaction radius (where they would overlap) increases with temperature. It is worth mentioning that the values found for both $R_{m}$ and $2 R_{\mathrm{HS}}$ agree with those obtained by Blundell when he inter-

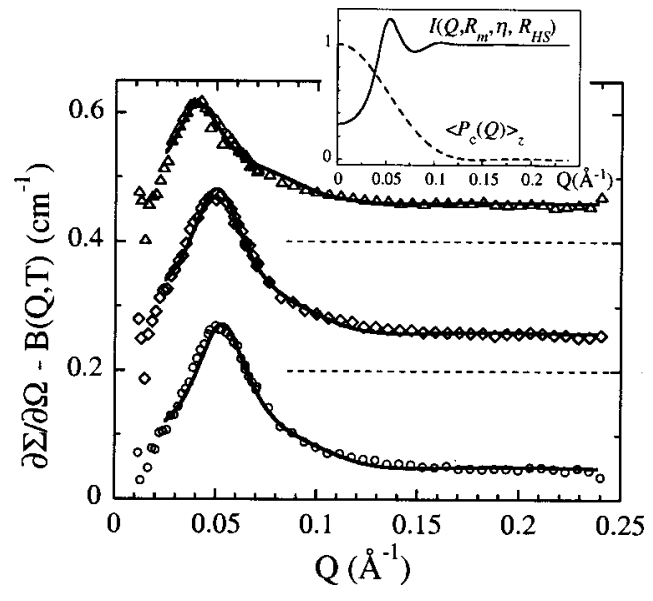

FIG. 10. Structure factor obtained for PVCd by SANS corrected for the low $Q$-decay at $360 \mathrm{~K}(\bigcirc), 430 \mathrm{~K}(\diamond)$, and $460 \mathrm{~K}(\triangle)$. The solid lines are the fitting curves obtained by applying the Percus-Yevick approach. The $430 \mathrm{~K}$ and the $460 \mathrm{~K}$ data have been shifted for clarity, being the new origins those marked by the dashed lines. The inset shows the two functions involved in the description of the $430 \mathrm{~K}$, namely, the interparticle structure factor $I\left(Q, R_{m}, \eta, R_{\mathrm{HS}}\right)$ (solid line) and the $z$-averaged intraparticle form factor $\left\langle P_{c}(Q)\right\rangle_{z}$ (dashed line) [see Eq. (19)].

preted SAXS measurements on PVC in terms of a nodular structure. ${ }^{3}$ On the other hand, we observe that the crystallinity $\phi_{c}$ decreases continuously from the constant value around $4 \%$ in the temperature range $360 \mathrm{~K} \leqslant T \leqslant 430 \mathrm{~K}$ to $\approx 1.6 \%$ at $470 \mathrm{~K}$. These values correspond also well to what is found usually in PVC (Ref. 1) and to recent NMR estimates in plasticized PVC. ${ }^{36}$ There, the plasticizer did not seem to effect the degree of crystallinity but merely acts to disperse the crystals.

In view of the rather featureless scattering curves obtained for PVCd-besides the peak structure- no further microscopic details have revealed themselves. The data, e.g., did not allow to distinguish Gaussian from log-normal distributions for the crystallite size. Nevertheless, from the SANS study of the PVCd and PVCd/PVCh samples we can extract some conclusions. The presented results corroborate the earlier invoked heterogeneity structure, and would not disagree with the scenario of irregularly packed nodules with a distribution of sizes proposed by Blundell. ${ }^{3}$ Moreover, our results on the chain form factor of PVC also support this scenario against the lamellar structure proposed by other authors. ${ }^{4}$ As we have seen in the previous section, the chains display a Gaussian conformation. The $\partial \Sigma / \partial \Omega(Q) \propto Q^{-2}$ dependence observed for the scattering from the $\mathrm{PVCd} / \mathrm{PVCh}$ blends at high $Q$-values excludes considerable sections of parallel stems as would be expected in long nodules. For such a structure a $Q^{-1}$ dependence of the scattered intensity should be obtained. The insert in Fig. 3 shows that our results are not compatible with a $Q^{-1}$ law. Moreover, it is very important to remark that our result for the chain dimension in the bulk $\left[R_{g_{w}}=(0.34 \pm 0.01) M_{w}^{0.5}\right]$ corresponds almost perfectly with the $\theta$-structure of PVC in Benzaldehyd at $430 \mathrm{~K}$ $\left(R_{g_{\theta}} / M_{w}^{0.5}=0.35\right) .{ }^{33}$ Taking into account the value for the main chain bond length in PVC $(1.53 \AA),{ }^{37}$ a characteristic ratio of $C_{\infty}=9.2 \pm 0.5$ for PVC chains in the bulk is derived from our study. This value agrees well with other values 
reported in the literature for the "unperturbed" chain; $C_{\infty}$ $=9.6-10.8$ (Ref. 38) and $C_{\infty}=10.9,{ }^{39}$ and is also compatible with estimations made using the PVC RIS model ${ }^{37}\left(C_{\infty}\right.$ $=11.8$ ). This again corroborates the Gaussian PVC chain conformations. Moreover, in contrast to the PVCd results, no peak has been found in the cross sections obtained for the PVCd/PVCh blends. We argue that the mean scattering length density of the inhomogeneity is very similar to that of the mean amorphous matrix due to the ideal mixing and zero Flory-Huggins coefficient. Thus, the contrast between these two components (crystalline and amorphous material) is much weaker than that between the deuterated chains and the protonated matrix, and the measurements in the blend reveal essentially the chain form factor without any hint of the inhomogeneities.

Summarizing, we may conclude that between $T_{g}$ and $430 \mathrm{~K}$ approximately the features of the heterogeneous structure of our sample do not change with temperature-apart from the usual thermal expansion. At higher temperatures a gradual structural homogeneization takes place. A completely homogeneous system would be obtained, if the modulation peak would have disappeared. An extrapolation of the integrated intensity [Fig. 2(c)] points to a temperature above $500 \mathrm{~K}$. This result agrees reasonably with the value estimated by Hoffman and Weeks ${ }^{40}$ for the maximum crystalline melting temperature $(542 \mathrm{~K})$.

\section{B. Heterogeneous dynamics}

The data analysis presented in the previous section reveals several anomalous features for PVC dynamics. First, in the neighborhood of $T_{g}$ the dielectric response attributable to the $\alpha$-relaxation cannot be described well by a single KWW function. The theoretical function provides too much intensity on the high frequency side and not enough at the low frequency flank of the loss peak compared to the experimental observation [see Fig. 4(b)]. In other words, close to $T_{g}$ the peak is unusually symmetric. On the other hand, in the dielectric study a strong $T$-dependence of the shape is observed, much steeper than that observed for polymers in general. ${ }^{41}$ Moreover, the value for the shape parameter $\beta$ in the neighborhood of $T_{g}$ is close to 0.3 , an extremely low value for polymers. Similar low values for this parameter have already been reported for PVC in the literature, like $\beta$ $=0.23,{ }^{26}$ and, as it was mentioned in the introduction, the broad response of PVC close to $T_{g}$ is very well known since a long time. ${ }^{12,13}$ However, a $T$-independent spectral shape was assumed in some previous works on PVC dynamics. ${ }^{26}$ The apparent discrepancies with these earlier results can be understood taking into account that the previous dielectric studies of PVC were restricted to a frequency range $5-10^{6} \mathrm{~Hz}$. If only such narrow interval is studied, the dielectric response can be rather well described by using an even broader theoretical function for the $\alpha$-process that fits the low frequency wing of the peak without dealing with the high frequency side. Moreover, the use of such a broad function allowed to fit the experimental data below $10^{6} \mathrm{~Hz}$ assuming values of $\kappa=1$ for the exponent in Eq. (6a). The extension of the experimental window towards higher fre- quencies (i) evidences the failure of the description used before in the new range accessed and (ii) shows that the value of $\kappa$ in Eq. (6a) has to be lower than 1.

The anomalies observed for the shape of the $\alpha$-process in PVC could be attributed to the merging process with the $\beta$-relaxation. ${ }^{16,42}$ This phenomenon could indeed contribute to the narrowing of the peaks at high temperatures-close to the merging of the timescales of the $\alpha$ - and $\beta$-relaxations. However, in the neighborhood of $T_{g}$ its influence should be weak, and never could produce the symmetrization observed for the $\alpha$-peak close to $T_{g}$.

Of great interest for a further interpretation are the similarities that can be found between the dynamical behavior of PVC and that of polymer blends. The dielectric response of a blend is characterized by a strong symmetrical broadening of the signal with respect to that of the homopolymer. ${ }^{43}$ This extra broadening rapidly decreases with increasing temperature. In such systems, the peculiar dynamical features find a natural explanation in the existence of different environments for the different regions in the material, i.e., in the presence of heterogeneities in the system. This analogy can be taken as a starting point for the interpretation of the PVC dynamical anomalies. As we have shown with the SANS study on the same sample, the presence of heterogeneities is evident. In our model of the PVC structure, a distribution of sizes was invoked. There are also indications in the literature that the noncrystalline portions may exist in various degrees of order with different densities. ${ }^{44}$ Density fluctuations associated to different packing degrees in our homopolymer would be the counterpart for concentration fluctuations in blends. It can be expected that regions with different density would show different dynamical behavior.

The presence of heterogeneities can also be deduced from the study of the $Q$-dependence of $S_{\text {inc }}(Q, t) .{ }^{29}$ In glassforming polymers the relationship of Eq. (17b) is usually found, implying Gaussian behavior for the incoherent scattering function. However, a coexistence of sample regions with different dynamics would cause deviations from Gaussianity. ${ }^{45}$ For example, for the case of polymer blends these deviations are well established. ${ }^{43} \mathrm{~A}$ weaker $Q$-dependence of the relaxation times than that given by Eq. (17b) can be observed in such materials. Also for PVC such a weaker $Q$-dependence is found (see Fig. 9).

All results point towards the existence of dynamical heterogeneities as the source of the anomalies observed. They would naturally originate from the structural heterogeneities found in our sample. In the following, we propose a model based on this hypothesis that rationalizes the dynamical behavior of PVC.

\section{Model of dynamical heterogeneities}

We start assuming the existence of a variety of regions in PVC which show different structural relaxations. The global response of the system is then given by the superposition of the individual responses.

We assume that within each region the $\alpha$ - or structural relaxation takes place in a similar way as observed for many polymers. We expect the $\alpha$-relaxation to follow a stretched exponential behavior with a dynamic structure factor accord- 
ing to Eq. (15), and to be associated to sublinear diffusion showing a Gaussian scattering function according to Eq. (18).

The spectral shape of the structural relaxation was determined by the NSE measurements at $Q_{\max }$, where the $\alpha$-relaxation is observed without contamination by secondary processes. ${ }^{16}$ Furthermore, at high enough temperatures the spectral shape was nearly $T$-independent and was described by a stretching exponent $\beta=0.5$, a reasonable value for a polymer. Also dielectric measurements in the same temperature range result in a similar $\beta$ value. Based on these observations, we make the assumption that the structural relaxation within any region is characterized by such a stretched exponential.

Thus, we consider a unique and to first order temperature independent spectral shape of the $\alpha$-relaxation for all regions; but we allow for different time scales in the different sample regions. We then suppose that the different regions are distributed by a log normal distribution. As a consequence, the characteristic time is distributed in the same way,

$$
g\left(\log \tau_{\alpha}\right)=\frac{1}{\sigma \sqrt{2 \pi}} \exp \left[-\frac{1}{2}\left(\frac{\log \tau_{\alpha}-\left\langle\log \tau_{\alpha}\right\rangle}{\sigma}\right)^{2}\right],
$$

where $\left\langle\log \tau_{\alpha}\right\rangle$ corresponds to the average time and $\sigma$ is the width of the distribution function. Both parameters can be $T$-dependent. The global relaxation observed by different experimental methods can be considered as a weighted integral over the local relaxations. Thus we define, independent of the experimental probe, a local correlator $f_{\alpha}\left(\tau_{\alpha}\right)$ with the above mentioned properties and a global correlator $F_{\alpha}$. Both are related by

$$
F_{\alpha}=\int_{0}^{\infty} g\left(\log \tau_{\alpha}\right) f_{\alpha}\left(\tau_{\alpha}\right) d\left(\log \tau_{\alpha}\right) .
$$

The relaxation times $\tau_{\alpha}$ stand for $\tau_{w}$ in Eq. (11), $\tau_{w}^{Q_{\max }}$ in Eq. (15) and $\tau_{0}$ in Eq. (18), which are all underlying the same Gaussian distribution.

Since the dielectric measurements cover the widest frequency/temperature range, the model was first applied to the permittivity data. There it was possible to accurately determine the parameters characterizing the distribution function. The analysis was carried out in a similar way as described in the previous section, i.e., taking into account the three contributions of Eq. (5) and fixing the $\beta$-relaxation function as determined from the sub- $T_{g}$ study. As we work in the frequency domain, the theoretical spectra were built by a superposition of $\mathrm{HN}$-functions equivalent to the KWW functions with $\beta=0.5$, i.e., with $\alpha=0.78, \gamma=0.55$. Using again a power law for the conductivity, the description of the spectra in the frame of this model was very satisfactory. For some of the temperatures this is demonstrated in Fig. 11. The assumed shape of the distribution function $g\left(\log \tau_{\alpha}\right)$ seems to be, at least within our experimental accuracy, appropriate to account for the observations, at least in the region $T$ $\geqslant 380 \mathrm{~K}$. At lower temperatures closer to $T_{g}$, some small discrepancies between model and experiment are present. However, the strong contribution of the conductivity in those spectra does not allow to decide whether $g\left(\log \tau_{\alpha}\right)$ becomes

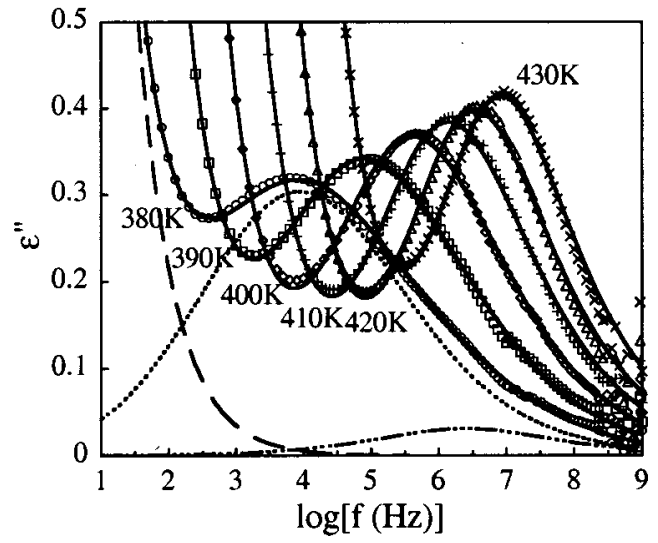

FIG. 11. Frequency dependence of the dielectric losses of PVC for the temperatures indicated. Points correspond to experimental data; solid lines to the fitting curves when the heterogeneous model is assumed. For $380 \mathrm{~K}$ the three contributions are shown: conductivity and interfacial polarization (dashed), $\beta$-relaxation (dashed-dotted), and $\alpha$-relaxation (dotted line).

asymmetric or not. The distribution functions $g\left(\log \tau_{w}\right)$ derived from the fits shown in Fig. 11 are depicted in Fig. 12(a). With increasing $T$ a clear narrowing of the distribution function is observed, and at high temperatures the timescales of the different regions become very similar. It is worth mentioning that this observation is in principle not related to structural changes, since the heterogeneous structure remains unaltered in the temperature range where $g\left(\log \tau_{w}\right)$ has been obtained. Figure 12(b) displays the associated strong decrease of the width $\sigma$ with increasing temperature. This $T$-dependence is well described by $\sigma=6.954-0.015 T[\mathrm{~K}]$ (solid line). Note that this parametrization of $\sigma$ leads to negative values at high temperatures; no distribution will be as-
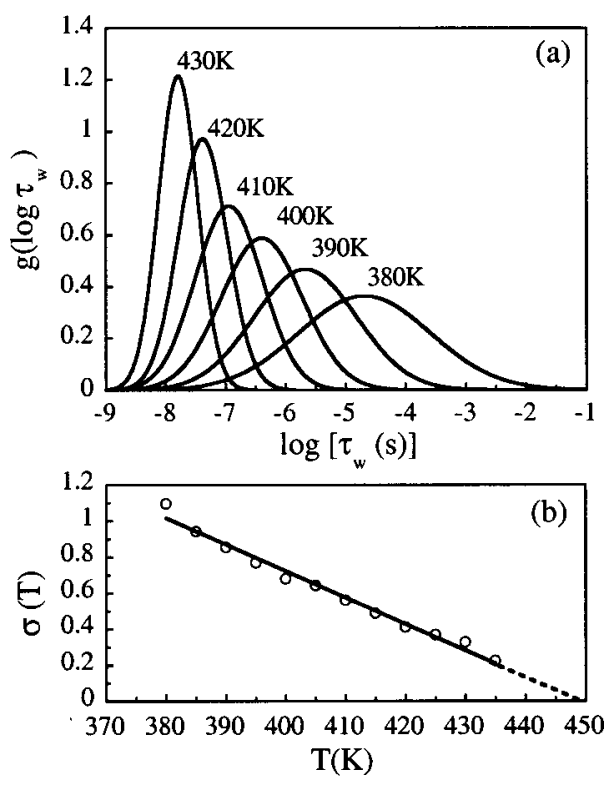

FIG. 12. (a) Distribution functions of KWW relaxation times as obtained from the description of the dielectric spectroscopy data with the heterogeneous model for the temperatures indicated. (b) Temperature dependence of the width of the distribution function. The solid line shows the fit of the points to the law proportional to $T$ given in the text, and the dashed line the extrapolation of this law to high temperatures. 


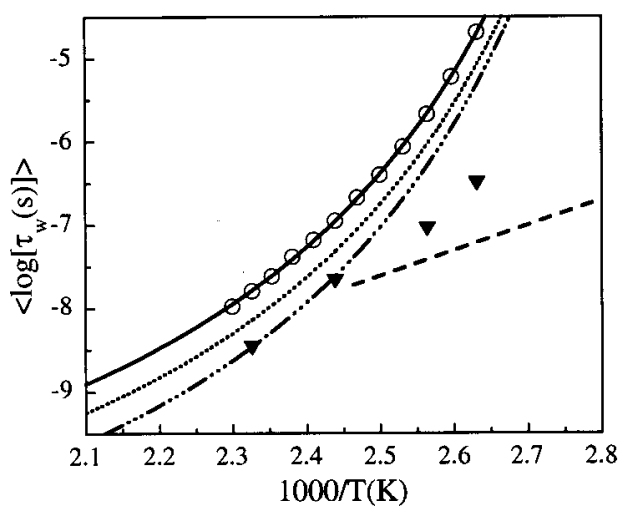

FIG. 13. Arrhenius plot of the average value of the distribution function $g\left(\log \tau_{w}\right)$ obtained from the description of the experimental data by the heterogeneous model. Symbols as in Fig. 6(b). Dotted and dashed-dotted lines are curves obtained by shifting the Vogel-Fulcher Law obtained from the dielectric spectroscopy results (solid line) in order to match the NSE results and the high temperature BS results at $1.2 \AA^{-1}$, respectively.

sumed in such cases. Finally, the $T$-dependence of the average relaxation time is depicted in Fig. 13. It can be very well described by a Vogel-Fulcher Law,

$$
\left\langle\log \tau_{\alpha}\right\rangle=\left\langle\log \tau_{\alpha}\right\rangle_{o}+\left(\frac{B \log e}{T-T_{o}}\right),
$$

with the values, $\left\langle\log \left[\tau_{w}(s)\right]\right\rangle_{o}=-11.66, B=1005.6 \mathrm{~K}$, and $T_{o}=317.24 \mathrm{~K}$.

On the other hand, the conductivity exponent $\kappa$ was determined to 0.8 , higher than that obtained in the former analysis. It has to be pointed out that it is not possible to describe properly the experimental behavior in the low frequency region by fixing $\kappa=1$. As commented above, this could indicate the presence of interfacial polarization processes contributing to $\varepsilon^{\prime \prime}(\omega)$ in the low frequency side. In fact, in the frame of our model such contribution would have a natural explanation in the heterogeneous character found for PVC.

We have shown that the proposed model accounts for the anomalous behavior of the dielectric response of PVC. The next step is to investigate whether the distribution function $g\left(\log \tau_{\alpha}\right)$ determined from the permittivity study also allows a description of the scattering data. In this way we also investigate the universality of the structural relaxation that is usually found in polymer systems. Universality means that the timescales for the different correlators show the same $T$-dependence. This feature would imply that the shift factor from the dielectric study allows to describe the experimental results obtained from other techniques.

We first discuss the data for coherent scattering from PVCd by NSE. The solid lines in Fig. 7 show the level of agreement achieved applying our model for $S\left(Q_{\max }, t\right) / S\left(Q_{\max }\right)$. An almost perfect description by the model is found. We note that the only parameters adjusted were the $T$-dependent parameter $A_{Q_{\max }}(T)$ accounting for fast processes and the prefactor $\left\langle\log \tau_{w}^{Q_{\max }}\right\rangle_{o}$ for the average value of the characteristic timescale of the structural relaxation. The resulting VF curve obtained for the average structural
TABLE II. Values obtained for the prefactor of the $\alpha$-relaxation in the dynamic structure factor by using the heterogeneous model.

\begin{tabular}{cc}
\hline \hline$T(\mathrm{~K})$ & $A_{Q_{\max }}$ \\
\hline 390 & 0.89 \\
400 & 0.88 \\
410 & 0.88 \\
430 & 0.87 \\
450 & 0.84 \\
460 & 0.82 \\
\hline
\end{tabular}

relaxation time is depicted in Fig. 13 with a dotted line. The value deduced for $\left\langle\log \left[\tau_{w}^{Q_{\max }}(\mathrm{s})\right]\right\rangle_{o}$ was -12.02 .

We note that due to the negligible width of $g\left(\log \tau_{\alpha}\right)$ at the highest temperatures, the curves obtained for $S\left(Q_{\max }, t\right) / S\left(Q_{\max }\right)$ are nearly indistinguishable from single KWW functions. However, as the temperature decreases towards $T_{g}$, the shape of the function imposed by $g\left(\log \tau_{\alpha}\right)$ and the $T$-dependence of $\left\langle\log \tau_{\alpha}\right\rangle$ perfectly account for the experimental behavior. This result is not trivial at all.

The values obtained for $A_{Q_{\max }}(T)$ are listed in Table II. They decrease continuously with increasing temperature, showing no signature for changing their behavior above a critical temperature $T_{c}$, as the Mode Coupling Theory ${ }^{46}$ would predict.

We now consider the incoherent scattering from PVCh. These data did not show the universality of the $\alpha$-process. For this reason at each temperature the spectra taken at different $Q$-values were fitted keeping the form of $g\left(\log \tau_{\alpha}\right)$ fixed to the dielectric result. A Debye-Waller factor like dependence of the prefactor $A(Q, T)$,

$$
A(Q, T)=A_{o} \exp \left[-\frac{\left\langle u^{2}\right\rangle}{3} Q^{2}\right],
$$

was imposed. Free parameters were $A_{o},\left\langle u^{2}\right\rangle$, and the mean value of the characteristic time distributed for this correlator, $\left\langle\log \tau_{o}\right\rangle$. They were determined as function of the temperature. The results for the parameters determining the amplitude of the incoherent spectra $A_{o}$ and $\left\langle u^{2}\right\rangle$ are listed in Table III. An acceptable description of the experimental spectra $S_{\text {inc }}(Q, \omega)$ was achieved, as can be appreciated in Fig. 8, validating again the shape used for $g\left(\log \tau_{\alpha}\right)$. It is worth noting that this distribution does not only determine the shape of the individual fitting curve for a given $Q$-value, but also the $Q$-dependence of the spectra. Let us remember that the time scale $\tau_{o}$ relates to the anomalous diffusion coefficient introduced above, and that the $Q$-dependence of the characteristic time $\tau_{w}(Q, T)$ in each region is required within our assumptions to follow a $Q^{-2 / \beta}(\beta=0.5)$ variation. This

TABLE III. Parameters determining the amplitude of the incoherent spectra by using the heterogeneous model.

\begin{tabular}{ccc}
\hline \hline$T(\mathrm{~K})$ & $A_{0}$ & $\left\langle u^{2}\right\rangle\left(\AA^{2}\right)$ \\
\hline 380 & 0.97 & 0.33 \\
390 & 0.96 & 0.34 \\
410 & 0.93 & 0.39 \\
430 & 0.89 & 0.35 \\
\hline \hline
\end{tabular}




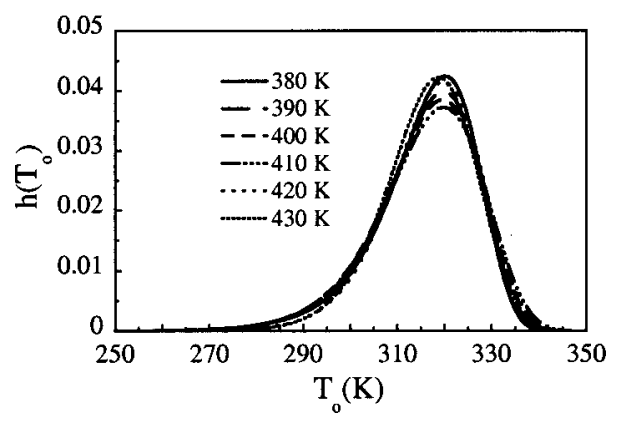

FIG. 14. Distribution functions of Vogel-Fulcher temperatures $T_{o}$ obtained from the distribution functions of KWW relaxation times $g\left(\log \tau_{w}\right)$ at the temperatures indicated.

is shown in Fig. 9, where the results obtained within this model for the average values of the timescales $\left\langle\log \tau_{w}\right\rangle$ are plotted as lines. These can easily be obtained from the values of $\left\langle\log \tau_{o}\right\rangle$ by means of Eq. (17) with $\beta=0.5$. In this figure, the vertical bars at some representative $Q$-values show the width of the distribution at the corresponding temperature. Note that the distribution can produce evident deviations from the Gaussian behavior, even at $430 \mathrm{~K}$, where $g\left(\log \tau_{\alpha}\right)$ is quite narrow (see the different slope of the times obtained from the single KWW description). The experimental behavior can thus be well reproduced by the superposition of diffusivities following $g\left(\log \tau_{o}\right)$.

In Fig. 13 the $T$-dependence of the average characteristic times such obtained from $S_{\text {inc }}(Q, t)$ is compared with the results from dielectric spectroscopy and coherent scattering. Both coherent and incoherent scattering data correspond to $Q=1.2 \AA^{-1}$. Deviations from universality are evident. At the two lowest temperatures the experimental behavior indicates a faster dynamics than that deduced for the $\alpha$-relaxation. To understand the origin of this observation, we examine the $Q$-range where the information has been obtained. Figure 9 shows with dots those $Q$-values where IN10 spectra are measured and where they exhibit some discernable broadening. The reliable $Q$-range for the lowest temperatures is reduced to values higher than $1 \AA^{-1}$. For other polymers like polybutadiene ${ }^{16}$ and polyisobutylene ${ }^{18}$ it was found that secondary relaxations cause quasielastic contributions of the spectra in this $Q$-regime. They dominate, if the $\alpha$-relaxation is too slow to contribute to the frequency window accessed by neutron scattering. Therefore, we can attribute part of the broadening observed in this $Q$ - and $T$-range to the $\beta$-process. This would explain the deviations from universality found in the analysis of $S_{\text {inc }}(Q, t)$. We note that the NSE results on PVCd represent a nice example for the isolation of the $\alpha$-relaxation by coherent measurements at $Q_{\max }$. In this case, no signature for deviations from the $T$-dependence of the characteristic time towards the $\beta$-process can be observed.

Figure 13 shows that the time scale for collective motion is slower than that of the self motion at the same $Q$-value $\left(Q_{\max }\right)$. This finding could indicate some modulation of the coherent times with $S(Q)$, in an analogous way to the predicted deGennes narrowing ${ }^{47}$ for simple liquids.

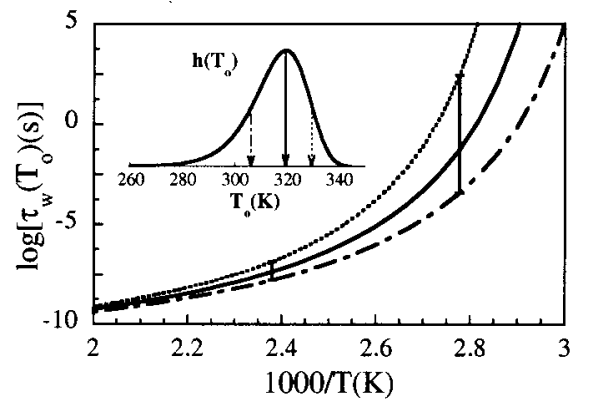

FIG. 15. Temperature dependence of the characteristic times of three different regions in the sample: those where $T_{o}$ correspond to the maximum (solid line) and half maximum (dotted and dashed-dotted lines) of the distribution function $h\left(T_{o}\right)$ (see insert). The vertical bars show the width of the distribution function of characteristic times $g\left(\log \tau_{w}\right)$ for two temperatures (360 $\mathrm{K}$ and $420 \mathrm{~K}$ ).

At this point we comment on a previous work featuring incoherent scattering results on PVC, that reported a very different $Q$-dependence for its characteristic time. ${ }^{26}$ Those results were obtained imposing a wrong shape parameter ( $\beta=0.23)$ on the fit of the quasielastic spectra which were of limited quality. The low $\beta$ value was deduced from the dielectric measurements restricted to $f \leqslant 10^{6} \mathrm{~Hz}$. From this wrong evaluation the importance of an extended frequency window in dielectric spectroscopy is evident. Furthermore, performing NSE investigations at the structure factor maximum is crucial for a determination of the actual shape of the relaxation function.

\section{Origin of the dynamical heterogeneities}

We have found a very satisfactory description of all sets of experimental results in terms of the heterogeneous model. The prior knowledge on the dynamics of polymer blends can guide us further in the interpretation of our results. Phenomenologically, for the poly(vinyl methyl ether) component dynamics in the poly(vinyl methyl ether)/polystyrene blends it has been found that the only parameter affected by blending is the VF temperature $T_{o} .{ }^{43}$ Thus, the distribution of relaxation times may be related to a distribution of VFtemperatures $h\left(T_{o}\right)$ in the sample. Note that a distribution of only $T_{o}$ translates in an equivalent distribution of glass transition temperatures. The question now is whether this also applies to the PVC heterogeneous behavior. If this holds, the distribution functions $g\left(\log \tau_{\alpha}\right)$ and $h\left(T_{o}\right)$ would be related through $g\left(\log \tau_{\alpha}\right) d\left(\log \tau_{\alpha}\right)=h\left(T_{o}\right) \mathrm{d} T_{o}$, and taking into account Eq. (22), $h\left(T_{o}\right)$ could be obtained from $g\left(\log \tau_{\alpha}\right)$,

$$
h\left(T_{o}\right)=g\left(\log \tau_{\alpha}\right) \frac{B \log e}{\left(T-T_{o}\right)^{2}} .
$$

In this way a unique function $h\left(T_{o}\right)$ is obtained from the different distribution functions $g\left(\log \tau_{\alpha}\right)$, independently of the temperature. This is demonstrated in Fig. 14. Within the uncertainties, all the curves collapse to one. This result corroborates the hypothesis that the behavior found is a consequence of the distribution of only the VF temperature. The resulting $h\left(T_{o}\right)$ shows a maximum around $320 \mathrm{~K}$, is asym- 
metric and can be considered as $T$-independent at least in the range $380 \mathrm{~K} \leqslant T \leqslant 430 \mathrm{~K}$ (see Fig. 14). Such properties are indeed what it would be expected from the structural study; SANS revealed a $T$-independent distribution of density domains in the same temperature range where $g\left(\log \tau_{\alpha}\right)$-and consequently $h\left(T_{o}\right)$ - has been determined. The temperature independence of the density domains demands temperature independent distributions $h\left(T_{o}\right)$. Let us note that, though the density modulations are hardly effected by the temperature in the $T$-range below $\approx 430 \mathrm{~K}$, the time scales of the different domains approach each other with increasing $T$. This leads to the observed narrowing of the time distribution function $g\left(\log \tau_{\alpha}\right)$ (see Fig. 15), which can be interpreted as a kind of "dynamical homogenization" of the system, but is not related to the structural changes.

At temperature above $430 \mathrm{~K}$, an additional homogenization of the dynamics related to the vanishing structural heterogeneities is expected. However, this effect cannot be resolved experimentally for the dynamics. This is due to the fact that the values for the variances of $g\left(\log \tau_{\alpha}\right)$ are already very small at such high temperatures. Nevertheless, we can conclude that the proposed scenario enables us to understand the dynamical anomalies of PVC in terms of its structural properties.

\section{CONCLUSIONS}

The combination of dielectric spectroscopy, coherent and incoherent neutron scattering for studying the dynamics, and SANS for resolving the heterogeneous structure of PVC, has allowed us to interpret the anomalous dynamical behavior of this polymer in terms of dynamical heterogeneities arising from the structural peculiarities. A distribution of glass transition temperatures due to the existence of density modulations in the sample causes the broadening of the response from the structural relaxation. The structural modulations persist in the temperature range $T<430 \mathrm{~K}$. In this regime, the system becomes dynamically homogeneous with increasing temperature, since the relaxation times of the different regions approach each other. At higher temperatures, a structural homogenization would additionally take place.

\section{ACKNOWLEDGMENTS}

The authors thank Professor C. Mijangos for the synthesis of the deuterated samples. A.M. acknowledges the grant from the University of the Basque Country, and A. A., A. A., and J.C. support from the following projects: DGICYT, PB97-0638; GV, EX 1998-23; UPV/EHU, 206.215-G20/98; 9/UPV 00206.215-13568/2001. Support from "Donostia International Physics Center" is also acknowledged.

\footnotetext{
${ }^{1}$ See, as a recent review, M. Gilbert, in Journal of Macromolecular Science Reviews in Macromolecular Chemistry and Physics C34, 77 (1994).

${ }^{2}$ See, as representative reference, Particulate Nature of $P V C$, edited by G. Butters (Applied Science, London, 1982).

${ }^{3}$ D. J. Blundell, Polymer 20, 934 (1979).

${ }^{4}$ W. Wenig, J. Polym. Sci., Polym. Phys. Ed. 16, 1635 (1978).

${ }^{5}$ D. J. Walsh, J. S. Higgins, C. P. Druke, and J. S. McKeown, Polymer 22, 168 (1981).

${ }^{6}$ D. G. H. Ballard, A. N. Burgess, J. M. Dekoninck, and E. A. Roberts, Polymer 28, 3 (1987).
}

${ }^{7}$ R. Scherrenberg, H. Reynaers, K. Mortensen, W. Vlak, and C. Gondard, Macromolecules 26, 3205 (1993).

${ }^{8}$ R. L. Scherrenberg, H. Reynaers, C. Gondard, and J. P. Verluyten, Macromolecules 26, 4118 (1993).

${ }^{9}$ R. L. Scherrenberg, H. Reynaers, C. Gondard, and P. A. M. Steeman, J. Polym. Sci., Part B: Polym. Phys. 32, 111 (1994).

${ }^{10}$ J. A. Juinj, G. H. Gisolf, and W. A. de Jong, Kolloid Z. Z. Polym. 251, 465 (1973).

${ }^{11}$ R. Hobson and A. H. Windle, Polymer 34, 3582 (1993).

${ }^{12}$ R. M. Fuoss and J. G. Kirkwood, J. Am. Chem. Soc. 63, 385 (1941).

${ }^{13}$ N. G. McCrum, B. E. Read, and G. Williams, in Anelastic and Dielectric Effects in Polymer Solids (Dover, New York, 1967).

${ }^{14}$ J. Colmenero, A. Alegría, J. M. Alberdi, F. Alvarez, and B. Frick, Phys. Rev. B 44, 7321 (1991).

${ }^{15}$ A. Arbe, U. Buchenau, L. Willner, D. Richter, B. Farago, and J. Colmenero, Phys. Rev. Lett. 76, 1872 (1996).

${ }^{16}$ A. Arbe, D. Richter, J. Colmenero, and B. Farago, Phys. Rev. E 54, 3853 (1996).

${ }^{17}$ D. Richter, A. Arbe, J. Colmenero, M. Monkenbusch, B. Farago, and R. Faust, Macromolecules 31, 1133 (1998).

${ }^{18}$ A. Arbe, J. Colmenero, B. Frick, M. Monkenbusch, and D. Richter, Macromolecules 31, 4926 (1998).

${ }^{19}$ See, e.g., C. Mijangos, G. Martínez, and J. Millán, Macromol. Chem., Macromol. Symp. 189, 567 (1988).

${ }^{20}$ P. A. Rogers, J. Appl. Polym. Sci. 48, 1061 (1993).

${ }^{21}$ Physical Properties of Polymers Handbook, edited by J. E. Mark (AIP, New York, 1996).

${ }^{22}$ See, e.g., Neutron Spin Echo, Lecture Notes in Physics, edited by F. Mezei (Springer-Verlag, Heidelberg, 1980), Vol. 28.

${ }^{23}$ J. Colmenero, A. Arbe, G. Coddens, B. Frick, C. Mijangos, and H. Reinecke, Phys. Rev. Lett. 78, 1928 (1997).

${ }^{24}$ F. Alvarez, A. Alegría, and J. Colmenero, Phys. Rev. B 44, 7306 (1991).

${ }^{25}$ F. Alvarez, A. Alegría, and J. Colmenero, Phys. Rev. B 47, 125 (1993).

${ }^{26}$ J. Colmenero, A. Alegría, A. Arbe, and B. Frick, Phys. Rev. Lett. 69, 478 (1992).

${ }^{27}$ J. Colmenero, A. Arbe, A. Alegría, and K. L. Ngai, J. Non-Cryst. Solids 172-174, 229 (1994).

${ }^{28}$ R. Zorn, Phys. Rev. B 55, 6249 (1997).

${ }^{29}$ A. Arbe, J. Colmenero, M. Monkenbusch, and D. Richter, Phys. Rev. Lett. 81, 590 (1998).

${ }^{30}$ B. Farago, A. Arbe, J. Colmenero, R. Faust, U. Buchenau, and D. Richter, Phys. Rev. E (in press, June 2002).

${ }^{31}$ A. Rahman, K. S. Singwi, and A. Sjölander, Phys. Rev. 126, 986 (1962).

${ }^{32}$ A. Arbe, A. Moral, J. Colmenero, W. Schmidt, and R. Kahn (unpublished).

${ }^{33}$ Polymer Handbook, 3rd ed., edited by J. Brandrup and E. H. Immergut (Wiley, New York, 1989).

${ }^{34}$ A. Crugnola, M. Pegoraro, and F. Danusso, J. Poly. Sci. Part A-Z 6, 1705 (1968).

${ }^{35}$ D. Kinning and E. Thomas, Macromolecules 17, 172 (1984).

${ }^{36}$ W. Barenswaard, V. Litvinov, F. Souren, R. Scherrenberg, C. Gondard, and C. Colemonts, Macromolecules 32, 167 (1999).

${ }^{37}$ P. J. Ludovice and U. W. Suter, in Computational Modeling of Polymers, edited by J. Bicerano (Marcel Dekker, New York, 1992).

${ }^{38}$ M. Sato, Y. Koshiishi, and M. Ashine, J. Polym. Sci., Part B: Polym. Lett. 1, 233 (1963).

${ }^{39}$ P. J. Ludovice, Doctoral thesis, M.I.T., January, 1989.

${ }^{40}$ J. D. Hoffman and J. Weeks, J. Res. Natl. Bur. Stand., Sect. A 66A, 13 (1962).

${ }^{41}$ A. Alegría, J. Colmenero, P. O. Mari, and I. A. Campbell, Phys. Rev. E 59, 6888 (1999).

${ }^{42}$ D. Gómez, A. Alegría, A. Arbe, and J. Colmenero, Macromolecules 34, 503 (2001).

${ }^{43}$ I. Cendoya, A. Alegría, J. M. Alberdi, J. Colmenero, H. Grimm, D. Richter, and B. Frick, Macromolecules 32, 4065 (1999).

${ }^{44}$ Yu. V. Glazkovskii, A. N. Zavyalov, N. M. Bakardzhiyer, and I. I. Novak, Polym. Sci. USSR 12, 3061 (1971).

${ }^{45}$ J. Colmenero, A. Arbe, A. Alegría, M. Monkenbusch, and D. Richter, J. Phys.: Condens. Matter 11, A363 (1999).

${ }^{46}$ See, e.g., W. Götze and L. Sjögren, Rep. Prog. Phys. 55, 241 (1992).

${ }^{47}$ P. G. deGennes, Physica (Amsterdam) 25, 128 (1959). 